\title{
Role of transverse momentum dependence of unpolarized parton distribution and fragmentation functions in the analysis of azimuthal spin asymmetries
}

\author{
M. Anselmino, ${ }^{1,2, *}$ M. Boglione, ${ }^{1,2, \dagger}$ U. D’Alesio, ${ }^{3,4, \sharp}$ F. Murgia, ${ }^{4, \S}$ and A. Prokudin ${ }^{5,6, \|}$ \\ ${ }^{1}$ Dipartimento di Fisica, Università di Torino, Via Pietro Giuria 1, I-10125 Torino, Italy \\ ${ }^{2}$ INFN, Sezione di Torino, Via Pietro Giuria 1, I-10125 Torino, Italy \\ ${ }^{3}$ Dipartimento di Fisica, Università di Cagliari, Cittadella Universitaria, I-09042 Monserrato (CA), Italy \\ ${ }^{4}$ INFN, Sezione di Cagliari, Cittadella Universitaria, I-09042 Monserrato (CA), Italy \\ ${ }^{5}$ Science Division, Penn State University Berks, Reading, Pennsylvania 19610, USA \\ ${ }^{6}$ Theory Center, Jefferson Lab, 12000 Jefferson Avenue, Newport News, Virginia 23606, USA
}

(Received 3 October 2018; published 27 November 2018)

\begin{abstract}
Information on the Sivers distribution and the Collins fragmentation functions and their transverse momentum dependence is mainly based on fitting single-spin asymmetry data from semi-inclusive deep inelastic scattering (SIDIS). Independent information on the Sivers distribution and the Collins fragmentation can be obtained from the Drell-Yan and $e^{+} e^{-}$annihilation processes, respectively. In the SIDIS case, the transverse momentum of the final observed hadron, which is the quantity measured, is generated both by the average transverse momentum in the distribution and by that in the fragmentation functions. As a consequence, these are strongly correlated, and a separate extraction is made difficult. In this paper we investigate, in a simple kinematical Gaussian configuration, this correlation, its role on the transverse single-spin asymmetries in SIDIS, and the consequences for predictions of the Sivers asymmetry in Drell-Yan processes and for the Collins asymmetry in $e^{+} e^{-}$annihilation. We find that, in some cases, these effects can be relevant and must be carefully taken into account.
\end{abstract}

DOI: 10.1103/PhysRevD.98.094023

\section{INTRODUCTION}

Transverse-momentum-dependent parton distribution and fragmentation functions (respectively, TMD PDFs and TMD FFs, collectively denoted as TMDs) are important tools for investigating the nucleon and its threedimensional structure. Among them, the Sivers function $[1,2]$ describes the asymmetry in the azimuthal distribution of unpolarized quarks and gluons around the direction of motion of a high-energy transversely polarized parent hadron. Similarly, the Collins fragmentation function [3] gives the azimuthal distribution of unpolarized hadrons around the direction of motion of a transversely polarized fragmenting quark. The former is related to the orbital motion of partons inside a nucleon, while the latter

\footnotetext{
*mauro.anselmino@to.infn.it

elena.boglione@to.infn.it

*umberto.dalesio@ca.infn.it

§francesco.murgia@ca.infn.it

"prokudin@jlab.org
}

Published by the American Physical Society under the terms of the Creative Commons Attribution 4.0 International license. Further distribution of this work must maintain attribution to the author(s) and the published article's title, journal citation, and DOI. Funded by SCOAP. describes fundamental properties of the hadronization process.

Azimuthal and transverse single-spin asymmetries (SSAs) in inclusive and semi-inclusive hadron production are the fundamental source of information on these nonperturbative functions. The Sivers and the Collins effects indeed play a crucial role in describing, within the so-called TMD factorization approach, many of the transverse and azimuthal asymmetries experimentally observed in semiinclusive deep inelastic scattering (SIDIS) and in $e^{+} e^{-}$ annihilations. The Sivers asymmetry is also crucial for understanding the single-spin asymmetries in polarized Drell-Yan processes, although experimental information in this case is still scarce. The transverse momentum dependence of the unpolarized TMDs is related to the $P_{T}$ distribution of hadrons produced in unpolarized SIDIS processes.

The first phase in the extraction of the TMDs from data can now be considered as complete. It has shown that the Sivers and Collins effects are indeed significant [4-14], and information on the Sivers and Collins functions is now available [15-23]. Using the Collins effect, the extraction of the quark transversity distribution has also been made possible [24]. In this phase a very simple parametrization of the unknown functions has been adopted, with factorized 
dependences on the different variables and a simple (and analytically integrable) Gaussian dependence on the transverse momenta. Thanks to important theoretical progress, a second phase has now started in which the QCD TMD evolution can be taken into account and a global fit of data from different processes can be attempted [25-32]. More refined and realistic parametrizations of the TMDs can be explored, leading to their more precise determination.

Before entering this phase, some considerations about the procedure of extraction of TMDs from data and the combined analysis of different processes are necessary. This concerns the way in which TMDs build up the measured quantities and the fact that often two of them are coupled into a unique observable; thus, disentangling information on a single TMD is not always straightforward and could lead to uncertainties which have to be taken into account. Here, we do this in the simple approach of the first phase mentioned above, which much simplifies and exemplifies the issues to be discussed, without spoiling their general features.

We start by noticing that most of the available information on spin asymmetries - related to the Sivers and Collins functions-and on unpolarized TMDs, is obtained from SIDIS processes data. In this case, however, the transverse momentum of the final observed hadron, $\boldsymbol{P}_{T}$, originates both from the transverse motion of the initial struck quark inside the nucleon, $\boldsymbol{k}_{\perp}$, and the transverse momentum of the final hadron with respect to the fragmenting quark, $\boldsymbol{p}_{\perp}$. At leading order in a $k_{\perp} / Q$ power expansion, where $Q$ is the hard scale for the process considered, one has

$$
\boldsymbol{P}_{T}=\boldsymbol{p}_{\perp}+z \boldsymbol{k}_{\perp},
$$

where $z$ is the light-cone momentum fraction of the hadron in the quark fragmentation process. As a consequence, in SIDIS, the transverse momentum dependences in the initial quark TMD PDFs and in the fragmenting quark TMD FFs are strongly correlated, as has been already pointed out $[26,27,31]$.

This dependence is usually parametrized by a Gaussian function, in which the main parameters are the widths $\left\langle k_{\perp}^{2}\right\rangle$ (for TMD PDFs) and $\left\langle p_{\perp}^{2}\right\rangle$ (for TMD FFs). Because of the relation given in Eq. (1), it is possible to obtain good fits of SIDIS data, with comparable $\chi_{\text {dof }}^{2}$, corresponding to different pairs of values for $\left\langle k_{\perp}^{2}\right\rangle$ and $\left\langle p_{\perp}^{2}\right\rangle$. However, the parameters of these comparable fits may lead to rather different consequences when used to get estimates for asymmetries in processes in which only TMD PDFs, like Drell-Yan processes, or only TMD FFs, like two-hadron production in $e^{+} e^{-}$annihilations, are involved.

In this paper we investigate this issue in more detail. To this end, we consider in the TMD factorization approach of the first phase the Sivers and Collins transverse single-spin asymmetries, together with the corresponding unpolarized cross sections, in SIDIS and Drell-Yan processes and in $e^{+} e^{-}$annihilations. The plan of the paper is the following:
In Sec. II, we will present the general expressions for the unpolarized cross sections and the single-spin asymmetries of interest for our study, referring to the original literature for their derivation. In Sec. III, we shall consider the study of the Sivers asymmetry in SIDIS and Drell-Yan processes, while in Sec. IV we will discuss the Collins asymmetries in SIDIS and $e^{+} e^{-}$annihilations. Finally, in Sec. V we will summarize our main results and their possible consequences for future studies of azimuthal and single-spin asymmetries in Drell-Yan processes and $e^{+} e^{-}$annihilations.

\section{GENERAL RESULTS IN THE TMD APPROACH}

In this section, we recall the formalism which we shall need for our discussion about the extraction of the transverse momentum dependence of the TMDs. In semiinclusive DIS, TMD factorization theorems [33-39] relate the transverse momentum of the produced hadron to the intrinsic transverse momenta of the parton both inside the target nucleon and in the quark hadronization process. Such factorization theorems, and the analogous ones for Drell-Yan processes and $e^{+} e^{-}$annihilations, are controllable approximations that allow one to relate the observed cross sections to convolutions of TMDs. Even though generic constraints on the functional form of the nonperturbative functions are given by the theorems themselves, a phenomenological analysis of the experimental data is needed to determine the functional shape of the TMDs.

We present the explicit expressions of the measured quantities within the TMD factorization approach at the parton model level and with Gaussian parametrizations for the TMDs; references to the original papers are given. It is, however, convenient to recall here the parametrizations adopted for the relevant TMDs. A parton inside a nucleon with momentum $\boldsymbol{P}$ has a momentum $\boldsymbol{p}=x \boldsymbol{P}+\boldsymbol{k}_{\perp}$, while a hadron produced in the fragmentation of a quark with momentum $\boldsymbol{p}_{q}$ has a momentum $\boldsymbol{P}_{h}=z \boldsymbol{p}_{q}+\boldsymbol{p}_{\perp}$. Notice that at leading order in a $k_{\perp} / Q$ power expansion, the longitudinal and light-cone momentum fractions coincide, neglecting the quark and final hadron masses.

The unpolarized TMD PDFs and TMD FFs are chosen, respectively, as [40]

$$
\begin{aligned}
f_{q / p}\left(x, k_{\perp}\right) & =f_{q / p}(x) \frac{e^{-k_{\perp}^{2} /\left\langle k_{\perp}^{2}\right\rangle}}{\pi\left\langle k_{\perp}^{2}\right\rangle}, \\
D_{h / q}\left(z, p_{\perp}\right) & =D_{h / q}(z) \frac{e^{-p_{\perp}^{2} /\left\langle p_{\perp}^{2}\right\rangle}}{\pi\left\langle p_{\perp}^{2}\right\rangle},
\end{aligned}
$$

while the Sivers function is written as

$$
\begin{aligned}
\Delta^{N} f_{q / p^{\uparrow}}\left(x, k_{\perp}\right) & =\Delta^{N} f_{q / p^{\uparrow}}(x) \sqrt{2 e} \frac{k_{\perp}}{M_{S}} e^{-k_{\perp}^{2} / M_{S}^{2}} \frac{e^{-k_{\perp}^{2} /\left\langle k_{\perp}^{2}\right\rangle}}{\pi\left\langle k_{\perp}^{2}\right\rangle} \\
& \equiv \Delta^{N} f_{q / p^{\uparrow}}(x) \sqrt{2 e} \frac{k_{\perp}}{M_{S}} \frac{e^{-k_{\perp}^{2} /\left\langle k_{\perp}^{2}\right\rangle}}{\pi\left\langle k_{\perp}^{2}\right\rangle}
\end{aligned}
$$


and the Collins function as

$$
\begin{aligned}
\Delta^{N} D_{h / q^{\uparrow}}\left(z, p_{\perp}\right) & =\Delta^{N} D_{h / q^{\uparrow}}(z) \sqrt{2 e} \frac{p_{\perp}}{M_{C}} e^{-p_{\perp}^{2} / M_{C}^{2}} \frac{e^{-p_{\perp}^{2} /\left\langle p_{\perp}^{2}\right\rangle}}{\pi\left\langle p_{\perp}^{2}\right\rangle} \\
& \equiv \Delta^{N} D_{h / q^{\uparrow}}(z) \sqrt{2 e} \frac{p_{\perp}}{M_{C}} \frac{e^{-p_{\perp}^{2} /\left\langle p_{\perp}^{2}\right\rangle_{C}}}{\pi\left\langle p_{\perp}^{2}\right\rangle}
\end{aligned}
$$

where we have defined

$$
\begin{aligned}
\left\langle k_{\perp}^{2}\right\rangle_{S} & =\frac{\left\langle k_{\perp}^{2}\right\rangle M_{S}^{2}}{\left\langle k_{\perp}^{2}\right\rangle+M_{S}^{2}}, \\
\left\langle p_{\perp}^{2}\right\rangle_{C} & =\frac{\left\langle p_{\perp}^{2}\right\rangle M_{C}^{2}}{\left\langle p_{\perp}^{2}\right\rangle+M_{C}^{2}} .
\end{aligned}
$$

These functional shapes are particularly suitable in order to directly impose the known positivity bounds on the Sivers and Collins functions. Notice that the factorized transverse momentum dependences have a Gaussian shape with a width which is constant and flavor independent.

\section{A. The Sivers SSA in the SIDIS process $\boldsymbol{\ell} \boldsymbol{p}^{\uparrow} \rightarrow \boldsymbol{\ell}^{\prime} \boldsymbol{h} \boldsymbol{X}$}

Following Ref. [40] (see also Ref. [41]), where all details can be found, the differential cross section for the semiinclusive production of a hadron $h$ in the current fragmentation region from the collision of an unpolarized lepton beam with a transversely polarized target can be written, in the deeply inelastic regime, as [see Eq. (79) of Ref. [40]]

$$
\begin{aligned}
\frac{\mathrm{d} \sigma^{\ell p\left(S_{T}\right) \rightarrow \ell^{\prime} h X}}{\mathrm{~d} x_{B} \mathrm{~d} Q^{2} \mathrm{~d} z_{h} \mathrm{~d}^{2} \boldsymbol{P}_{T} \mathrm{~d} \phi_{S}}= & \frac{2 \alpha^{2}}{Q^{4}}\left\{\frac{1+(1-y)^{2}}{2} F_{U U}+\cdots\right. \\
& \left.+\left[\frac{1+(1-y)^{2}}{2} \sin \left(\phi_{h}-\phi_{S}\right) F_{U T}^{\sin \left(\phi_{h}-\phi_{S}\right)}+(1-y) \sin \left(\phi_{h}+\phi_{S}\right) F_{U T}^{\sin \left(\phi_{h}+\phi_{S}\right)}+\cdots\right]\right\} .
\end{aligned}
$$

We have considered the case of a transversely polarized $\operatorname{target}\left(S_{T}=1, S_{L}=0\right)$ and an unpolarized beam $\left(P_{z}^{\ell}=0\right)$; we have omitted terms which are not related to the Sivers or Collins asymmetries. $x_{B}, y, z_{h}$, and $Q$ are the usual SIDIS variables. Notice that, at order $k_{\perp} / Q, x_{B}=x$ and $z_{h}=z$. $P_{T}$ is the magnitude of the hadron transverse momentum in the $\gamma *$-nucleon center-of-mass frame; $\phi_{h}$ and $\phi_{S}$ are, respectively, the azimuthal angle of the observed hadron and that of the target polarization vector with respect to the leptonic plane. The subscript $U T$ in the structure functions $F$ reminds us that we are considering the case of an unpolarized lepton beam and a transversely polarized nucleon target ( $U U$ refers to the unpolarized situation).

In the SIDIS case, the asymmetries are expressed through their azimuthal moments:

$$
A_{U T}^{W\left(\phi_{h}, \phi_{S}\right)}=2 \frac{\int d \phi_{h} d \phi_{S}\left[d \sigma^{\uparrow}-d \sigma^{\downarrow}\right] W\left(\phi_{h}, \phi_{S}\right)}{\int d \phi_{h} d \phi_{S}\left[d \sigma^{\uparrow}+d \sigma^{\downarrow}\right]},
$$

where $W\left(\phi_{h}, \phi_{S}\right)$ is the appropriate azimuthal weight function required in order to isolate the specific contribution of interest and $d \sigma^{\uparrow, \downarrow}$ is the differential cross section of Eq. (6), with $S_{T}=\uparrow, \downarrow$ denoting a transverse polarization with the azimuthal angles $\phi_{S}$ and $\phi_{S}+\pi$, respectively. Then we simply have

$$
\begin{aligned}
d \sigma^{\uparrow}-d \sigma^{\downarrow}= & \frac{2 \alpha^{2}}{Q^{4}}\left\{\left[1+(1-y)^{2}\right] \sin \left(\phi_{h}-\phi_{S}\right) F_{U T}^{\sin \left(\phi_{h}-\phi_{S}\right)}\right. \\
& \left.+2(1-y) \sin \left(\phi_{h}+\phi_{S}\right) F_{U T}^{\sin \left(\phi_{h}+\phi_{S}\right)}+\cdots\right\},
\end{aligned}
$$

$$
d \sigma^{\uparrow}+d \sigma^{\downarrow}=\frac{2 \alpha^{2}}{Q^{4}}\left\{\left[1+(1-y)^{2}\right] F_{U U}+\cdots\right\} .
$$

The Sivers asymmetry is related to the $\sin \left(\phi_{h}-\phi_{S}\right)$ modulation, and from Eqs. (7)-(9) we find

$$
A_{U T}^{\sin \left(\phi_{h}-\phi_{S}\right)}=\frac{F_{U T}^{\sin \left(\phi_{h}-\phi_{S}\right)}}{F_{U U}} .
$$

From Eqs. (115) and (123) of Ref. [40] (remember that $x_{B}=x$ and $\left.z_{h}=z\right)$, we see that

$$
\begin{aligned}
& F_{U U}=\sum_{q} e_{q}^{2} f_{q / p}(x) D_{h / q}(z) \frac{e^{-P_{T}^{2} /\left\langle P_{T}^{2}\right\rangle}}{\pi\left\langle P_{T}^{2}\right\rangle}, \\
& F_{U T}^{\sin \left(\phi_{h}-\phi_{S}\right)}= \sum_{q} e_{q}^{2} \Delta^{N} f_{q / p^{\uparrow}}(x) D_{h / q}(z) \\
& \times \sqrt{\frac{e}{2}} \frac{P_{T}}{M_{S}} \frac{z\left\langle k_{\perp}^{2}\right\rangle_{S}^{2}}{\left\langle k_{\perp}^{2}\right\rangle} \frac{e^{-P_{T}^{2} /\left\langle P_{T}^{2}\right\rangle_{S}}}{\pi\left\langle P_{T}^{2}\right\rangle_{S}^{2}},
\end{aligned}
$$

where [see Eq. (131) of Ref. [40]]

$$
\left\langle P_{T}^{2}\right\rangle=\left\langle p_{\perp}^{2}\right\rangle+z^{2}\left\langle k_{\perp}^{2}\right\rangle, \quad\left\langle P_{T}^{2}\right\rangle_{S}=\left\langle p_{\perp}^{2}\right\rangle+z^{2}\left\langle k_{\perp}^{2}\right\rangle_{S},
$$

with $\left\langle k_{\perp}^{2}\right\rangle_{S}$ as in Eq. (5).

These relations, valid at first order in a $k_{\perp} / Q$ power expansion, show explicitly the strong correlation in building the physical observables between the properties of the 
partonic transverse momentum distribution and those of the partonic fragmentation. It is, in fact, the analysis of these entangled effects which motivates our study. Notice that this correlation is also modulated by the value of $z$.

From Eqs. (10)-(12) we see that the Sivers azimuthal asymmetry for SIDIS processes can be factorized as

$$
A_{U T}^{\sin \left(\phi_{h}-\phi_{S}\right)}\left(x, z, P_{T}\right)=A_{\mathrm{DIS}}^{S}(x, z) F_{\mathrm{DIS}}^{S}\left(z, P_{T}\right),
$$

where

$$
\begin{gathered}
A_{\mathrm{DIS}}^{S}(x, z)=\frac{\sum_{q} e_{q}^{2} \Delta^{N} f_{q / p^{\uparrow}}(x) D_{h / q}(z)}{2 \sum_{q} e_{q}^{2} f_{q / p}(x) D_{h / q}(z)}, \\
F_{\mathrm{DIS}}^{S}\left(z, P_{T}\right)=\frac{\sqrt{2 e} \frac{P_{T}}{M_{S}} \frac{z\left\langle k_{\perp}^{2}\right\rangle_{S}^{2} \exp \left[-P_{T}^{2} /\left\langle P_{T}^{2}\right\rangle_{S}\right]}{\pi\left\langle k_{\perp}^{2}\right\rangle\left\langle P_{T}^{2}\right\rangle_{S}^{2}}}{\frac{\exp \left[-P_{T}^{2} /\left\langle P_{T}^{2}\right\rangle\right]}{\pi\left\langle P_{T}^{2}\right\rangle}} .
\end{gathered}
$$

If we now integrate separately the numerator and the denominator of $F_{\mathrm{DIS}}^{S}$ over the modulus of the transverse momentum of the observed hadron, $P_{T} d P_{T}$, in the full $P_{T}$ range $[0,+\infty)$, and define the dimensionless parameters

$$
\xi_{1}=\frac{\left\langle p_{\perp}^{2}\right\rangle}{\left\langle k_{\perp}^{2}\right\rangle}, \quad \rho_{S}=\frac{\left\langle k_{\perp}^{2}\right\rangle_{S}}{\left\langle k_{\perp}^{2}\right\rangle}=\frac{M_{S}^{2}}{M_{S}^{2}+\left\langle k_{\perp}^{2}\right\rangle},
$$

we find the $P_{T}$-integrated Sivers asymmetry for SIDIS,

$$
A_{U T}^{\sin \left(\phi_{h}-\phi_{S}\right)}(x, z)=A_{\mathrm{DIS}}^{S}(x, z) \mathcal{F}_{\mathrm{DIS}}^{S}(z),
$$

where

$$
\mathcal{F}_{\text {DIS }}^{S}\left(z, \xi_{1}, \rho_{S}\right)=\sqrt{\frac{e \pi}{2}}\left[\frac{\rho_{S}^{3}\left(1-\rho_{S}\right)}{\rho_{S}+\xi_{1} / z^{2}}\right]^{1 / 2} .
$$

Notice that $0<\rho_{S}<1$. For $M_{S}^{2} \ll\left\langle k_{\perp}^{2}\right\rangle, \rho_{S} \rightarrow 0$; in this case, the $k_{\perp}$-dependent part of the Sivers function is sharply peaked around zero, and at its maximum almost equals the $k_{\perp}$-dependent component of the unpolarized distribution. On the other hand, for $M_{S}^{2} \gg\left\langle k_{\perp}^{2}\right\rangle, \rho_{S} \rightarrow 1$; correspondingly, the $k_{\perp}$-dependent part of the Sivers function is peaked around $\sqrt{\left\langle k_{\perp}^{2}\right\rangle / 2}$, where its value becomes smaller and smaller. Both these borderline cases are not very relevant from the phenomenological point of view, although for completeness we shall consider the full range of values for $\rho_{S}$.

A comment on the $P_{T} d P_{T}$ integration, which applies as well to the sections following, is necessary. Such an integration can be performed analytically and leads to very simple results, but it exceeds the range of validity of the TMD factorization, which holds up to transverse momenta of the order of a few GeVonly, such that $P_{T} / z \ll Q$. Above that, higher-order QCD corrections become dominant. However, because of the Gaussian dependences, the large
$P_{T}$ values do not contribute significantly to the integrations, which are indeed dominated by the region of validity of the TMD factorization. Our fully $P_{T}$-integrated expressions can be safely compared with data collected at small $P_{T}$ values $\left(P_{T}\right.$ up to $\left.1-2 \mathrm{GeV}\right)$.

\section{B. The Collins SSA in SIDIS processes}

The Collins effect generates a $\sin \left(\phi_{h}+\phi_{S}\right)$ modulation, and from Eqs. (7), (8), and (9), we find that the azimuthal moment of the Collins asymmetry in SIDIS processes can be written as

$$
A_{U T}^{\sin \left(\phi_{h}+\phi_{S}\right)}=\frac{2(1-y)}{1+(1-y)^{2}} \frac{F_{U T}^{\sin \left(\phi_{h}+\phi_{S}\right)}}{F_{U U}},
$$

where $F_{U U}$ is given by Eq. (11) and $F_{U T}^{\sin \left(\phi_{h}+\phi_{S}\right)}$ can be taken from Eq. (127) of Ref. [40] (noticing that the parameter $M_{h}$ is here denoted as $M_{C}$ ):

$$
\begin{aligned}
F_{U T}^{\sin \left(\phi_{h}+\phi_{S}\right)}= & \sum_{q} e_{q}^{2} h_{1}^{q}(x) \Delta^{N} D_{h / q^{\uparrow}}(z) \\
& \times \sqrt{\frac{e}{2}} \frac{P_{T}}{M_{C}} \frac{\left\langle p_{\perp}^{2}\right\rangle_{C}^{2}}{\left\langle p_{\perp}^{2}\right\rangle} \frac{e^{-P_{T}^{2} /\left\langle P_{T}^{2}\right\rangle_{T}}}{\pi\left\langle P_{T}^{2}\right\rangle_{T}^{2}} .
\end{aligned}
$$

In this equation, $h_{1}^{q}(x)$ is the $k_{\perp}$-integrated, collinear quark transversity distribution, $\Delta^{N} D_{h / q^{\uparrow}}(z)$ is the z-dependent term in the Collins fragmentation function [see Eq. (4)], $\left\langle k_{\perp}^{2}\right\rangle_{T}$ is the flavor-independent average square transverse momentum for the transversity distribution, and

$$
\left\langle P_{T}^{2}\right\rangle_{T}=\left\langle p_{\perp}^{2}\right\rangle_{C}+z^{2}\left\langle k_{\perp}^{2}\right\rangle_{T},
$$

with $\left\langle p_{\perp}^{2}\right\rangle_{C}$ given in Eq. (5).

In complete analogy to the Sivers asymmetry, in the Collins case also we can write

$A_{U T}^{\sin \left(\phi_{h}+\phi_{S}\right)}\left(x, y, z, P_{T}\right)=A_{\text {DIS }}^{C}(x, y, z) F_{\text {DIS }}^{C}\left(z, P_{T}\right)$,

where

$A_{\mathrm{DIS}}^{C}(x, y, z)=\frac{1-y}{1+(1-y)^{2}} \frac{\sum_{q} e_{q}^{2} h_{1}^{q}(x) \Delta^{N} D_{h / q^{\uparrow}}(z)}{\sum_{q} e_{q}^{2} f_{q / p}(x) D_{h / q}(z)}$,

$$
F_{\text {DIS }}^{C}\left(z, P_{T}\right)=\frac{\sqrt{2 e} \frac{P_{T}}{M_{C}} \frac{\left\langle p_{\perp}^{2}\right\rangle_{C}^{2} \exp \left[-P_{T}^{2} /\left\langle P_{T}^{2}\right\rangle_{T}\right]}{\pi\left\langle p_{\perp}^{2}\right\rangle\left\langle P_{T}^{2}\right\rangle_{T}^{2}}}{\frac{\exp \left[-P_{T}^{2} /\left\langle P_{T}^{2}\right\rangle\right]}{\pi\left\langle P_{T}^{2}\right\rangle}} .
$$

Once more, by integrating separately the numerator and denominator of $F_{\text {DIS }}^{C}$ over $P_{T} d P_{T}$ in the full range $[0,+\infty)$, and defining the dimensionless parameters

$$
\xi_{T}=\frac{\left\langle k_{\perp}^{2}\right\rangle_{T}}{\left\langle k_{\perp}^{2}\right\rangle}, \quad \rho_{C}=\frac{\left\langle p_{\perp}^{2}\right\rangle_{C}}{\left\langle p_{\perp}^{2}\right\rangle}=\frac{M_{C}^{2}}{M_{C}^{2}+\left\langle p_{\perp}^{2}\right\rangle},
$$


we can write the $P_{T}$-integrated Collins asymmetry for SIDIS as

$$
A_{U T}^{\sin \left(\phi_{h}+\phi_{S}\right)}(x, y, z)=A_{\text {DIS }}^{C}(x, y, z) \mathcal{F}_{\text {DIS }}^{C}(z),
$$

with

$$
\mathcal{F}_{\mathrm{DIS}}^{C}\left(z, \rho_{C}, \xi_{1} / \xi_{T}\right)=\sqrt{\frac{e \pi}{2}}\left[\frac{\rho_{C}^{3}\left(1-\rho_{C}\right)}{\rho_{C}+z^{2}\left(\xi_{T} / \xi_{1}\right)}\right]^{1 / 2} .
$$

Notice the similarity with $\mathcal{F}_{\text {DIS }}^{S}$ in Eq. (19).

\section{The Sivers SSA in Drell-Yan processes,$$
h_{1}^{\uparrow} h_{2} \rightarrow \ell^{+} \ell^{-} X
$$

Similarly to the SIDIS case, the Sivers asymmetry to be measured in DY processes is (see Ref. [42] for all details)

$$
\begin{aligned}
A_{N}^{\sin \left(\phi_{\gamma}-\phi_{S}\right)} & \equiv A_{N}^{\mathrm{DY}}\left(y, M, q_{T}\right) \\
& =2 \frac{\int d \phi_{\gamma}\left[d \sigma^{\uparrow}-d \sigma^{\downarrow}\right] \sin \left(\phi_{\gamma}-\phi_{S}\right)}{\int d \phi_{\gamma}\left[d \sigma^{\uparrow}+d \sigma^{\downarrow}\right]},
\end{aligned}
$$

where $d \sigma^{\uparrow, \downarrow}$ stands here for the cross section

$$
\frac{d^{4} \sigma^{h_{1}^{\uparrow, \downarrow} h_{2} \rightarrow \ell^{+} \ell^{-} X}}{d y d M^{2} d^{2} \boldsymbol{q}_{T}},
$$

with $y, M$, and $\boldsymbol{q}_{T}$ being the rapidity, the invariant mass, and the transverse momentum of the final leptonic pair, respectively, while $\phi_{\gamma}$ and $\phi_{S}$ are the azimuthal angle of the virtual boson and of the transverse polarization of the initial hadron in the center-of-mass frame of the two colliding hadrons, respectively.

We limit our discussion to the energy regime $M \ll M_{W, Z}$, where electromagnetic contributions dominate, neglecting weak interaction terms. Following Ref. [42], with the parametrization of the TMDs as in Eqs. (2) and (3), the numerator and the denominator of the SSA $A_{N}^{\mathrm{DY}}$ read

$$
\begin{aligned}
\operatorname{Num}\left[A_{N}^{\mathrm{DY}}\right]= & \frac{4 \pi \alpha^{2}}{9 M^{2} s} \sum_{q} e_{q}^{2} \Delta^{N} f_{q / h_{1}^{\uparrow}}\left(x_{1}\right) f_{\bar{q} / h_{2}}\left(x_{2}\right) \sqrt{2 e} \frac{q_{T}}{M_{S}} \\
& \times \frac{\left\langle k_{\perp}^{2}\right\rangle_{S}^{2} \exp \left[-q_{T}^{2} /\left(\left\langle k_{\perp}^{2}\right\rangle_{S}+\left\langle k_{\perp 2}^{2}\right\rangle\right)\right]}{\pi\left\langle k_{\perp 1}^{2}\right\rangle\left[\left\langle k_{\perp}^{2}\right\rangle_{S}+\left\langle k_{\perp 2}^{2}\right\rangle\right]^{2}} \\
\operatorname{Den}\left[A_{N}^{\mathrm{DY}}\right]= & \frac{4 \pi \alpha^{2}}{9 M^{2} s} 2 \sum_{q} e_{q}^{2} f_{q / h_{1}}\left(x_{1}\right) f_{\bar{q} / h_{2}}\left(x_{2}\right) \\
& \times \frac{\exp \left[-q_{T}^{2} /\left(\left\langle k_{\perp 1}^{2}\right\rangle+\left\langle k_{\perp 2}^{2}\right\rangle\right)\right]}{\pi\left[\left\langle k_{\perp 1}^{2}\right\rangle+\left\langle k_{\perp 2}^{2}\right\rangle\right]}
\end{aligned}
$$

Here $x_{1}$ and $x_{2}$ are, as usual, the light-cone momentum fractions of the active quark and antiquark annihilating into the final lepton pair; $\left\langle k_{\perp 1}^{2}\right\rangle$ and $\left\langle k_{\perp 2}^{2}\right\rangle$ are the average square transverse momenta of the unpolarized quarks or antiquarks inside the unpolarized initial hadrons. They are taken to be flavor and $x_{1,2}$ independent. In general, they can be different for different hadrons, like, for example, in the pion-proton DY processes measured at COMPASS. At leading order in a $k_{\perp} / M$ power expansion, as is well known, one has

$$
x_{1}=\frac{M}{\sqrt{s}} e^{y}, \quad x_{2}=\frac{M}{\sqrt{s}} e^{-y} .
$$

Again, the Sivers SSA $A_{N}^{\mathrm{DY}}$ factorizes into two terms, one $\left(x_{1}, x_{2}\right)$ dependent and one $q_{T}$ dependent:

$$
A_{N}^{\mathrm{DY}}\left(y, M, q_{T}\right)=A_{\mathrm{DY}}^{S}\left(x_{1}, x_{2}\right) F_{\mathrm{DY}}^{S}\left(q_{T}\right),
$$

where

$$
\begin{gathered}
A_{\mathrm{DY}}^{S}\left(x_{1}, x_{2}\right) \equiv A_{\mathrm{DY}}^{S}(y, M)=\frac{\sum_{q} e_{q}^{2} \Delta^{N} f_{q / h_{1}^{\uparrow}}\left(x_{1}\right) f_{\bar{q} / h_{2}}\left(x_{2}\right)}{2 \sum_{q} e_{q}^{2} f_{q / h_{1}}\left(x_{1}\right) f_{\bar{q} / h_{2}}\left(x_{2}\right)}, \\
F_{\mathrm{DY}}^{S}\left(q_{T}\right)=\frac{\sqrt{2 e} \frac{q_{T}}{M_{S}} \frac{\left\langle k_{\perp}^{2}\right\rangle_{S}^{2} \exp \left[-q_{T}^{2} /\left(\left\langle k_{\perp}^{2}\right\rangle_{S}+\left\langle k_{\perp 2}^{2}\right\rangle\right)\right]}{\pi\left\langle k_{\perp 1}^{2}\right\rangle\left[\left\langle k_{\perp}^{2}\right\rangle_{S}+\left\langle k_{\perp 2}^{2}\right\rangle\right]^{2}}}{\frac{\exp \left[-q_{T}^{2} /\left(\left\langle k_{\perp 1}^{2}\right\rangle+\left\langle k_{\perp 2}^{2}\right\rangle\right)\right]}{\pi\left[\left\langle k_{\perp 1}^{2}\right\rangle+\left\langle k_{\perp 2}^{2}\right\rangle\right]}} .
\end{gathered}
$$

By integrating separately the numerator and denominator of $F_{\mathrm{DY}}^{S}$ over $q_{T} d q_{T}$ in the full range $[0,+\infty)$ and defining the dimensionless parameters

$$
\xi_{21}=\frac{\left\langle k_{\perp 2}^{2}\right\rangle}{\left\langle k_{\perp 1}^{2}\right\rangle}, \quad \rho_{S}=\frac{\left\langle k_{\perp}^{2}\right\rangle_{S}}{\left\langle k_{\perp 1}^{2}\right\rangle}=\frac{M_{S}^{2}}{M_{S}^{2}+\left\langle k_{\perp 1}^{2}\right\rangle},
$$

we get the $P_{T}$-integrated Sivers asymmetry for DY:

$$
A_{N}^{\mathrm{DY}}(y, M)=A_{\mathrm{DY}}^{S}\left(x_{1}, x_{2}\right) \mathcal{F}_{\mathrm{DY}}^{S},
$$

with the simple expression

$$
\mathcal{F}_{\mathrm{DY}}^{S}\left(\rho_{S}, \xi_{21}\right)=\sqrt{\frac{e \pi}{2}}\left[\frac{\rho_{S}^{3}\left(1-\rho_{S}\right)}{\rho_{S}+\xi_{21}}\right]^{1 / 2} .
$$

Notice the similarity between $\mathcal{F}_{\text {DY }}^{S}$ and $\mathcal{F}_{\text {DIS }}^{S}$ in Eq. (19).

\section{The Collins azimuthal asymmetry in $e^{+} e^{-} \rightarrow h_{1} h_{2} X$ processes}

Finally, we consider the Collins azimuthal asymmetry for two almost back-to-back hadrons produced in opposite jets in $e^{+} e^{-}$annihilations. We do this in the so-called hadronic plane method, which is the most reliable from an experimental point of view, since it does not require the reconstruction of the jet thrust axis. On the other hand, from a theoretical point of view, this method explicitly requires 
the assumption of a factorized, Gaussian-shaped transverse momentum dependence in the fragmentation functions. The adoption of the thrust axis method, which is somehow more clean theoretically, would lead to similar results and conclusions.

In the hadronic plane kinematical configuration, one measures the transverse momentum $\boldsymbol{P}_{1 T}$ of the first hadron $h_{1}$, with respect to the plane containing the initial lepton beams and the second hadron $h_{2}$. Following Ref. [21] and references therein, the differential cross section for the process under consideration can be written as

$\frac{d \sigma^{e^{+} e^{-} \rightarrow h_{1} h_{2} X}}{d z_{1} d z_{2} d^{2} \boldsymbol{P}_{1 T} d \cos \theta}=\frac{3 \pi \alpha^{2}}{2 s}\left\{D_{h_{1} h_{2}}+N_{h_{1} h_{2}} \cos \left(2 \phi_{1}\right)\right\}$,

where $\theta$ is the angle between the direction of motion of $h_{2}$ and the beam axis; $\phi_{1}$ is the azimuthal angle of $\boldsymbol{P}_{1 T}$; and $z_{1,2}$ are the light-cone momentum fractions of the hadrons $h_{1,2}$.

From Eqs. (30) and (31) of Ref. [21], we have

$$
\begin{aligned}
D_{h_{1} h_{2}}= & \left(1+\cos ^{2} \theta\right) \sum_{q} e_{q}^{2} D_{h_{1} / q}\left(z_{1}\right) D_{h_{2} / \bar{q}}\left(z_{2}\right) \\
& \times \frac{\exp \left[-P_{1 T}^{2} /\left\langle\tilde{p}_{\perp}^{2}\right\rangle\right]}{\pi\left\langle\tilde{p}_{\perp}^{2}\right\rangle} \\
N_{h_{1} h_{2}}= & \frac{1}{4} \frac{z_{1} z_{2}}{z_{1}^{2}+z_{2}^{2}} \sin ^{2} \theta \sum_{q} e_{q}^{2} \Delta^{N} D_{h_{1} / q^{\uparrow}}\left(z_{1}\right) \Delta^{N} D_{h_{2} / \bar{q}^{\uparrow}}\left(z_{2}\right) \\
& \times \frac{2 e P_{1 T}^{2}}{\left\langle\tilde{p}_{\perp}^{2}\right\rangle+\tilde{M}_{C}^{2}} \frac{\exp \left[-P_{1 T}^{2}\left(\frac{1}{\tilde{M}_{C}^{2}}+\frac{1}{\left\langle\tilde{p}_{\perp}^{2}\right\rangle}\right)\right]}{\pi\left\langle\tilde{p}_{\perp}^{2}\right\rangle}
\end{aligned}
$$

where

$$
\tilde{M}_{C}^{2}=\frac{z_{1}^{2}+z_{2}^{2}}{z_{2}^{2}} M_{C}^{2}, \quad\left\langle\tilde{p}_{\perp}^{2}\right\rangle=\frac{z_{1}^{2}+z_{2}^{2}}{z_{2}^{2}}\left\langle p_{\perp}^{2}\right\rangle,
$$

and $M_{C}$ is the parameter introduced in the Collins function in Eqs. (4) and (5). Notice that the factorized $z$-dependent part of the Collins function, $\Delta^{N} D_{h / q^{\uparrow}}(z)$, was denoted $\tilde{\Delta}^{N} D_{h / q^{\uparrow}}(z)$ in Ref. [21].

For simplicity, we are assuming that $h_{1}$ and $h_{2}$ are both either pions or kaons, leaving aside, for instance, the $\pi K$ case that would in general require two different $\left\langle p_{\perp}^{2}\right\rangle$ values.

The azimuthal asymmetries of interest are the $\cos \left(2 \phi_{1}\right)$ modulations of the cross section in Eq. (40), driven by the ratios $N_{h_{1} h_{2}} / D_{h_{1} h_{2}}$. Data have been taken for different charge combinations of the two hadrons-that is, $h_{1} h_{2}=$ $\pi^{+} \pi^{-}+\pi^{-} \pi^{+}(U), \pi^{+} \pi^{+}+\pi^{-} \pi^{-}(L)$, and $\pi^{+} \pi^{-}+\pi^{-} \pi^{+}+$ $\pi^{+} \pi^{+}+\pi^{-} \pi^{-}(C)$. The actual quantities measured are

$$
A_{0}^{U L(C)} \simeq P_{0}^{U}-P_{0}^{L(C)},
$$

where

$$
P_{0}^{U, L, C}=\frac{N_{h_{1} h_{2}}^{U, L, C}}{D_{h_{1} h_{2}}^{U, L, C}} .
$$

From Eqs. (41) and (42), we can write

$P_{0}^{h_{1} h_{2}}\left(z_{1}, z_{2}, P_{1 T} ; \theta\right)=A_{\mathrm{ee}}^{h_{1} h_{2}}\left(z_{1}, z_{2} ; \theta\right) F_{\mathrm{ee}}^{C}\left(z_{1}, z_{2}, P_{1 T}\right)$,

where

$$
\begin{aligned}
A_{\mathrm{ee}}^{h_{1} h_{2}}\left(z_{1}, z_{2} ; \theta\right)= & \frac{1}{4} \frac{\sin ^{2} \theta}{1+\cos ^{2} \theta} \frac{z_{1} z_{2}}{z_{1}^{2}+z_{2}^{2}} \\
& \times \frac{\sum_{q} e_{q}^{2} \Delta^{N} D_{h_{1} / q^{\uparrow}}\left(z_{1}\right) \Delta^{N} D_{h_{2} / \bar{q}^{\uparrow}}\left(z_{2}\right)}{\sum_{q} e_{q}^{2} D_{h_{1} / q}\left(z_{1}\right) D_{h_{2} / \bar{q}}\left(z_{2}\right)}, \\
F_{\mathrm{ee}}^{C}\left(z_{1}, z_{2}, P_{1 T}\right)= & \frac{\frac{2 e P_{1 T}^{2}}{\left\langle\tilde{p}_{\perp}^{2}\right\rangle+\tilde{M}_{C}^{2}} \frac{\exp \left[-P_{1 T}^{2}\left(\frac{1}{\tilde{M}_{C}^{2}}+\frac{1}{\left\langle\tilde{p}_{\perp}^{2}\right\rangle}\right)\right]}{\pi\left\langle\tilde{p}_{\perp}^{2}\right\rangle}}{\frac{\exp \left[-P_{1 T}^{2} /\left\langle\tilde{p}_{\perp}^{2}\right\rangle\right]}{\pi\left\langle\tilde{p}_{\perp}^{2}\right\rangle}} .
\end{aligned}
$$

Once again, we proceed by integrating separately the numerator and denominator of $F_{\text {ee }}^{C}$ over $P_{1 T} d P_{1 T}$ in the full range $[0,+\infty)$, finding

$$
P_{0}^{h_{1} h_{2}}\left(z_{1}, z_{2} ; \theta\right)=A_{\mathrm{ee}}^{h_{1} h_{2}}\left(z_{1}, z_{2} ; \theta\right) \mathcal{F}_{\mathrm{ee}}^{C},
$$

with

$$
\mathcal{F}_{\text {ee }}^{C}\left(\rho_{C}\right)=2 e \rho_{C}^{2}\left(1-\rho_{C}\right) .
$$

Notice that $\mathcal{F}_{\text {ee }}^{C}$ is independent of $z_{1}$ and $z_{2}$.

\section{E. Summary of main formulas}

It is convenient to collect here, all together, the main results of the previous sections, which will be used in the sections to follow.

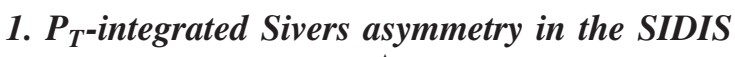
process $\ell_{p^{\uparrow}} \rightarrow \ell^{\prime} h X$

$$
\begin{gathered}
A_{U T}^{\sin \left(\phi_{h}-\phi_{S}\right)}(x, z)=A_{\mathrm{DIS}}^{S}(x, z) \mathcal{F}_{\mathrm{DIS}}^{S}(z), \\
A_{\mathrm{DIS}}^{S}(x, z) \text { as in Eq. }(15),
\end{gathered}
$$

$$
\begin{aligned}
\mathcal{F}_{\mathrm{DIS}}^{S}\left(z, \rho_{S}, \xi_{1}\right) & =\sqrt{\frac{e \pi}{2}}\left[\frac{\rho_{S}^{3}\left(1-\rho_{S}\right)}{\rho_{S}+\xi_{1} / z^{2}}\right]^{1 / 2}, \quad \xi_{1}=\frac{\left\langle p_{\perp}^{2}\right\rangle}{\left\langle k_{\perp}^{2}\right\rangle} \\
\rho_{S} & =\frac{\left\langle k_{\perp}^{2}\right\rangle_{S}}{\left\langle k_{\perp}^{2}\right\rangle}=\frac{1}{1+\frac{\left\langle k_{\perp}^{2}\right\rangle}{M_{S}^{2}}} .
\end{aligned}
$$




\section{2. $P_{T}$-integrated Collins asymmetry} in the SIDIS process $\ell_{p^{\uparrow}} \rightarrow \ell^{\prime} h X$

$$
\begin{aligned}
A_{U T}^{\sin \left(\phi_{h}+\phi_{S}\right)}(x, y, z) & =A_{\text {DIS }}^{C}(x, y, z) \mathcal{F}_{\text {DIS }}^{C}(z), \\
A_{\text {DIS }}^{C}(x, z) & \text { as in Eq. }(24), \\
\mathcal{F}_{\text {DIS }}^{C}\left(z, \rho_{C}, \xi_{1} / \xi_{T}\right) & =\sqrt{\frac{e \pi}{2}}\left[\frac{\rho_{C}^{3}\left(1-\rho_{C}\right)}{\rho_{C}+z^{2}\left(\xi_{T} / \xi_{1}\right)}\right]^{1 / 2}, \\
\xi_{T} & =\frac{\left\langle k_{\perp}^{2}\right\rangle_{T}}{\left\langle k_{\perp}^{2}\right\rangle}, \quad \rho_{C}=\frac{\left\langle p_{\perp}^{2}\right\rangle_{C}}{\left\langle p_{\perp}^{2}\right\rangle}=\frac{1}{1+\frac{\left\langle p_{\perp}^{2}\right\rangle}{M_{C}^{2}}} .
\end{aligned}
$$

$$
\begin{gathered}
\text { 3. } \boldsymbol{q}_{T^{-i n t e g r a t e d ~ S i v e r s ~ a s y m m e t r y ~}} \\
\text { in the DY process, } \boldsymbol{h}_{\mathbf{1}}^{\uparrow} \boldsymbol{h}_{\mathbf{2}} \rightarrow \boldsymbol{\ell}^{+\boldsymbol{+}} \boldsymbol{e}^{-\boldsymbol{X}} \\
A_{N}^{\mathrm{DY}}(y, M)=A_{\mathrm{DY}}^{S}\left(x_{1}, x_{2}\right) \mathcal{F}_{\mathrm{DY}}^{S}, \\
A_{\mathrm{DY}}^{S}\left(x_{1}, x_{2}\right) \text { as in Eq. }(35), \\
\mathcal{F}_{\mathrm{DY}}^{S}\left(\rho_{S}, \xi_{21}\right)=\sqrt{\frac{e \pi}{2}}\left[\frac{\rho_{S}^{3}\left(1-\rho_{S}\right)}{\rho_{S}+\xi_{21}}\right]^{1 / 2}, \quad \xi_{21}=\frac{\left\langle k_{\perp 2}^{2}\right\rangle}{\left\langle k_{\perp 1}^{2}\right\rangle}, \\
\rho_{S}=\frac{\left\langle k_{\perp}^{2}\right\rangle_{S}}{\left\langle k_{\perp 1}^{2}\right\rangle}=\frac{1}{1+\frac{\left\langle k_{\perp 1}^{2}\right\rangle}{M_{S}^{2}}} . \\
\text { 4. } \boldsymbol{P}_{T^{-} \text {-integrated } \text { Collins asymmetry }^{2}} \text { in the process } \boldsymbol{e}^{+} \boldsymbol{e}^{-} \rightarrow \boldsymbol{h}_{\mathbf{1}} \boldsymbol{h}_{\mathbf{2}} \boldsymbol{X} \\
P_{0}^{h_{1} h_{2}}\left(z_{1}, z_{2} ; \theta\right)=A_{\mathrm{ee}}^{h_{1} h_{2}}\left(z_{1}, z_{2} ; \theta\right) \mathcal{F}_{\mathrm{ee}}^{C}, \\
A_{\mathrm{ee}}^{h_{1} h_{2}}\left(z_{1}, z_{2} ; \theta\right) \text { as in Eq. }(47), \\
\mathcal{F}_{\mathrm{ee}}^{C}\left(\rho_{C}\right)=2 e \rho_{C}^{2}\left(1-\rho_{C}\right) .
\end{gathered}
$$

\section{4. $P_{T}$-integrated Collins asymmetry}

\section{THE SIVERS CASE}

In this section, we consider the fit of the Sivers SSAs in SIDIS and Drell-Yan processes, and the possible phenomenological uncertainties induced by the strong correlation between $\left\langle k_{\perp}^{2}\right\rangle$ and $\left\langle p_{\perp}^{2}\right\rangle$ in SIDIS azimuthal asymmetries in Eqs. (1) and (13). More precisely, since at present only a few experimental results are available on the Sivers SSA in Drell-Yan processes, we study the consequences for predictions on this observable due to the uncertainty on $\left\langle k_{\perp}^{2}\right\rangle$ and $\left\langle p_{\perp}^{2}\right\rangle$ as extracted from SIDIS data.

According to the present experimental situation, the amount of available SIDIS data on the Sivers azimuthal asymmetry $A_{U T}^{\sin \left(\phi_{h}-\phi_{S}\right)}$ allows us to obtain a sufficiently well-constrained parametrization of the quark Sivers distributions, $\Delta f_{q / p^{\uparrow}}(x)$, at least in some kinematical ranges (the present SIDIS data are limited to the $x_{\mathrm{B}} \lesssim 0.3$ region). We denote by $\hat{\rho}_{S}$ and $\hat{\xi}_{1}$ the particular values of $\rho_{S}$ and $\xi_{1}$ in Eq. (52), corresponding to a SIDIS best fit of the Sivers function. We shall adopt the "hat" symbol also for the corresponding Sivers SSAs.

Notice that for Drell-Yan processes with two different initial beams, as is the case for the COMPASS experiment at CERN, where one considers the reaction $\pi p^{\uparrow} \rightarrow \ell^{+} \ell^{-} X$, one should also take into account the parameter $\xi_{21}$ introduced in the previous sections. In order to simplify the analysis and focus on the main issue, we only consider the case $\xi_{21}=1$, corresponding to $p p$ collisions.

As mentioned in the Introduction, different studies of unpolarized azimuthal distributions [43], hadron multiplicities [26,27,31], and the Sivers SSA in SIDIS processes have been performed. These studies have indeed shown a strong correlation between $\left\langle k_{\perp}^{2}\right\rangle$ and $\left\langle p_{\perp}^{2}\right\rangle$, which manifests itself in large differences in the values of $\xi_{1}=\left\langle p_{\perp}^{2}\right\rangle /\left\langle k_{\perp}^{2}\right\rangle$, which can be associated with different, equally good fits of the same quantities-in particular, the Sivers asymmetry $A_{U T}^{\sin \left(\phi_{h}-\phi_{S}\right)}$.

To be definite, we consider in particular two different parametrization sets for the Sivers distributions, which lead to comparable values of $\chi_{\text {dof }}^{2}$ :

(1) The fit of Ref. [17], referred to as FIT09, for which

$$
\begin{aligned}
\left\langle k_{\perp}^{2}\right\rangle & =0.25 \mathrm{GeV}^{2}, \quad\left\langle p_{\perp}^{2}\right\rangle=0.20 \mathrm{GeV}^{2}, \\
M_{S}^{2} & =0.34 \mathrm{GeV}^{2},
\end{aligned}
$$

implying

$$
\hat{\xi}_{1}^{(09)}=0.80, \quad \hat{\rho}_{S}^{(09)}=0.58 .
$$

The complete list of parameters fixing the Sivers functions can be found in Table I of Ref. [17], where more details on the fitting procedure, the parameter extraction, and additional references are given.

It is important to remember here that, for this as well as for all the following reference fits adopted, the values of $\left\langle k_{\perp}^{2}\right\rangle$ and $\left\langle p_{\perp}^{2}\right\rangle$ are first extracted from observables depending only on the unpolarized TMD distribution and fragmentation functions, and then used, as fixed parameters, in the fitting procedure of the azimuthal spin asymmetries.

(2) The fit from Ref. [23], referred to as FIT16, for which

$$
\begin{aligned}
\left\langle k_{\perp}^{2}\right\rangle & =0.57 \mathrm{GeV}^{2}, \quad\left\langle p_{\perp}^{2}\right\rangle=0.12 \mathrm{GeV}^{2}, \\
M_{S}^{2} & =0.80 \mathrm{GeV}^{2},
\end{aligned}
$$

implying

$$
\hat{\xi}_{1}^{(16)}=0.21, \quad \hat{\rho}_{S}^{(16)}=0.58 .
$$

Again, detailed information and the complete list of parameters can be found in Ref. [23] and its Table I.

Notice that the two parametrizations show very different values of $\hat{\xi}_{1}$ but almost identical values of $\hat{\rho}_{S}$. This has the 
consequence that $\hat{\mathcal{F}}_{\mathrm{DY}}^{S} \equiv \mathcal{F}_{\mathrm{DY}}^{S}\left(\hat{\rho}_{S}, \xi_{21}=1\right)$ is the same for the two reference fits, FIT09 and FIT16.

The possibility of obtaining equally good fits of the SIDIS Sivers data with different values of the parametersin particular, $\xi_{1}$ - can be formalized by assuming that, at least in some limited regions of the $\left(\rho_{S}, \xi_{1}\right)$ parameter space, in moving away from the reference point along some trajectory, one keeps having

$$
A_{\mathrm{DIS}}^{S}(x, z) \mathcal{F}_{\mathrm{DIS}}^{S}\left(z, \rho_{S}, \xi_{1}\right) \simeq \hat{A}_{\mathrm{DIS}}^{S}(x, z) \hat{\mathcal{F}}_{\mathrm{DIS}}^{S}\left(z, \hat{\rho}_{S}, \hat{\xi}_{1}\right) .
$$

Notice that by changing the values of $\xi_{1}$ and $\rho_{S}$, one obtains in general different values of $\mathcal{F}_{\text {DIS }}^{S}\left(z, \rho_{S}, \xi_{1}\right)$ : then, by fitting the same data either with the lhs or the rhs of Eq. (63), one extracts different values of $\Delta f_{q / p^{\uparrow}}(x)$, which is contained in $A_{\mathrm{DIS}}^{S}$ in Eq. (15). In fact, one has

$$
A_{\mathrm{DIS}}^{S} \simeq\left(\frac{\hat{\mathcal{F}}_{\mathrm{DIS}}^{S}}{\mathcal{F}_{\mathrm{DIS}}^{S}}\right) \hat{A}_{\mathrm{DIS}}^{S} .
$$

The predictions for the DY Sivers asymmetry, made using the SIDIS Sivers function $\Delta f_{q / p^{\uparrow}}(x)$, are then affected by its uncertainty; as both $A_{\mathrm{DIS}}^{S}$ and $A_{\mathrm{DY}}^{S}$ are linear in the Sivers function, it is natural to assume that

$$
\frac{A_{\mathrm{DY}}^{S}}{\hat{A}_{\mathrm{DY}}^{S}} \simeq \frac{A_{\mathrm{DIS}}^{S}}{\hat{A}_{\mathrm{DIS}}^{S}},
$$

which, using Eq. (64), implies

$$
A_{\mathrm{DY}}^{S} \simeq\left(\frac{\hat{\mathcal{F}}_{\mathrm{DIS}}^{S}}{\mathcal{F}_{\mathrm{DIS}}^{S}}\right) \hat{A}_{\mathrm{DY}}^{S} .
$$

Notice that from Eqs. (52) and (64), one has

$$
\mathcal{F}_{\mathrm{DIS}}^{S}=R_{\mathrm{DIS}}^{S} \hat{\mathcal{F}}_{\mathrm{DIS}}^{S}, \quad A_{\mathrm{DIS}}^{S} \simeq \frac{1}{R_{\mathrm{DIS}}^{S}} \hat{A}_{\mathrm{DIS}}^{S},
$$

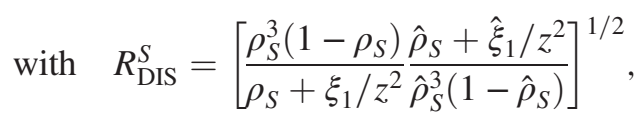

and, analogously, from Eq. (56) with $\xi_{21}=1$ and Eq. (66), one has

$$
\begin{aligned}
& \mathcal{F}_{\mathrm{DY}}^{S}=R_{\mathrm{DY}}^{S} \hat{\mathcal{F}}_{\mathrm{DY}}^{S}, \quad A_{\mathrm{DY}}^{S} \simeq \frac{1}{R_{\mathrm{DIS}}^{S}} \hat{A}_{\mathrm{DY}}^{S}, \quad \text { with } \\
& R_{\mathrm{DY}}^{S}=\left[\frac{\rho_{S}^{3}\left(1-\rho_{S}\right)}{\rho_{S}+1} \frac{\hat{\rho}_{S}+1}{\hat{\rho}_{S}^{3}\left(1-\hat{\rho}_{S}\right)}\right]^{1 / 2} .
\end{aligned}
$$

Then, when moving in the parameter space from $\left(\hat{\rho}_{S}, \hat{\xi}_{1}\right)$ to $\left(\rho_{S}, \xi_{1}\right)$ along a generic trajectory, the predictions for the Sivers DY asymmetry change as

$$
A_{N}^{\mathrm{DY}}=A_{\mathrm{DY}}^{S} \mathcal{F}_{\mathrm{DY}}^{S} \simeq\left(\frac{R_{\mathrm{DY}}^{S}}{R_{\mathrm{DIS}}^{S}}\right) \hat{A}_{\mathrm{DY}}^{S} \hat{\mathcal{F}}_{\mathrm{DY}}^{S}=R_{\mathrm{DY}}^{N} \hat{A}_{N}^{\mathrm{DY}},
$$

where

$$
R_{\mathrm{DY}}^{N}=\left[\frac{\rho_{S}+\xi_{1} / z^{2}}{\hat{\rho}_{S}+\hat{\xi}_{1} / z^{2}} \frac{\hat{\rho}_{S}+1}{\rho_{S}+1}\right]^{1 / 2} .
$$

Let us now discuss some possible different scenarios, one corresponding to the parameters of the sets FIT09 and FIT16 $\left(R_{\mathrm{DY}}^{S}=1\right)$, and two more exploratory cases $\left(R_{\mathrm{DIS}}^{S}=1\right.$ and $\left.R_{\mathrm{DY}}^{N}=1\right)$.

\section{A. Sivers effect, scenario 1: FIT09 vs FIT16}

This is the case which motivated our study. We have two different parametrization sets of the Sivers distribution, FIT09 and FIT16 discussed above, which describe comparably well the Sivers azimuthal asymmetry measured in SIDIS processes. We have investigated to what extent the corresponding estimates for the Sivers asymmetry in DrellYan processes can differ due to the uncertainty on the $\xi_{1}$ parameter, see Eqs. (60) and (62). Notice that, in this case, $\hat{\rho}_{S}^{(09)}=\hat{\rho}_{S}^{(16)} \equiv \hat{\rho}_{S}$.

From Eq. (68), then, one sees that $R_{\mathrm{DY}}^{S}=1$ (remember that we are considering the case of $p p$ collisions here-that is, $\xi_{21}=1$ ), and from Eqs. (69) and (70), one obtains that, going from one set of parameters to the other, the predictions for $A_{N}^{\mathrm{DY}}$ are rescaled as

$$
A_{N}^{\mathrm{DY}}\left(\hat{\rho}_{S}, \hat{\xi}_{1}^{(16)}\right) \simeq\left[\frac{\hat{\rho}_{S}+\hat{\xi}_{1}^{(16)} / z^{2}}{\hat{\rho}_{S}+\hat{\xi}_{1}^{(09)} / z^{2}}\right]^{1 / 2} \hat{A}_{N}^{\mathrm{DY}}\left(\hat{\rho}_{S}, \hat{\xi}_{1}^{(09)}\right) .
$$

Using the values given in Eqs. (60) and (62), one sees that the rescaling factor in the above equation varies from about 0.52 to 0.68 for $z$ in the range [0.1, 0.7]. Since small $z$ values dominate the SIDIS data, we find that

$$
A_{N}^{\mathrm{DY}}\left(\hat{\rho}_{S}, \hat{\xi}_{1}^{(16)}\right) \simeq \frac{1}{2} \hat{A}_{N}^{\mathrm{DY}}\left(\hat{\rho}_{S}, \hat{\xi}_{1}^{(09)}\right)
$$

This simple example, based on two available fits of the quark Sivers function, clearly shows how the uncertainty in the parameter $\xi_{1}=\left\langle p_{\perp}^{2}\right\rangle /\left\langle k_{\perp}^{2}\right\rangle$, due to the unavoidable strong correlation between $\left\langle k_{\perp}^{2}\right\rangle$ and $\left\langle p_{\perp}^{2}\right\rangle$ in SIDIS processes, see Eq. (13), induces large differences when trying to estimate the Sivers SSA in Drell-Yan processes. This effect should be carefully taken into account when studying these asymmetries and their related fundamental properties, e.g., the TMD scale evolution of the Sivers function and its process dependence.

\section{B. Sivers effect, scenario 2: Fixing $A_{\text {DIS }}^{S}$ and $\mathcal{F}_{\text {DIS }}^{S}$}

In the previous scenario, based on the fact that two equally good fits of the Sivers SIDIS asymmetry, FIT09 and FIT16, yield the same values of $\rho_{S}$ even if one starts with very different values of $\xi_{1}$, we have shown how the corresponding predictions for the Sivers asymmetries in 

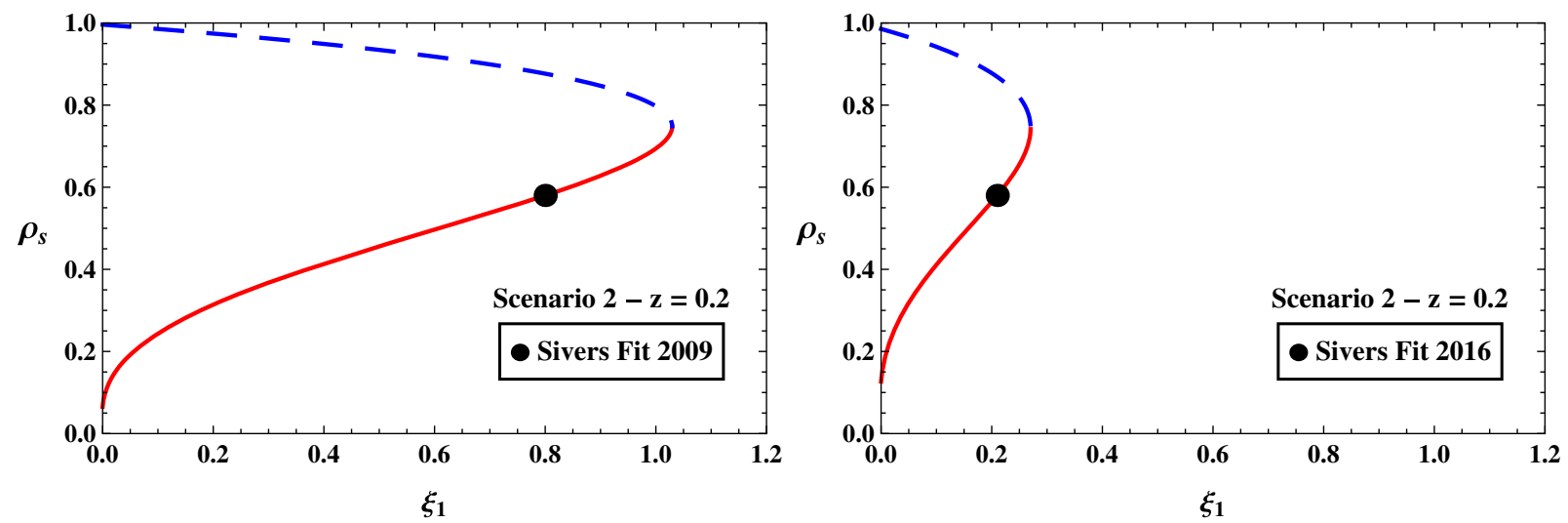

FIG. 1. The curves in the $\left(\rho_{S}, \xi_{1}\right)$ parameter space show the set of values of $\rho_{S}$ and $\xi_{1}$ which leave unchanged the $P_{T}$-integrated factor of the Sivers asymmetry, $\mathcal{F}_{\text {DIS }}^{S}(z=0.2)$. The black dots correspond to the values $\hat{\rho}_{S}$ and $\hat{\xi}_{1}$ obtained in the fits of Ref. [17] (left plot, FIT09) and of Ref. [23] (right plot, FIT16), which describe equally well the SIDIS Sivers asymmetry. Notice that for each value of $\xi_{1}$ one finds two possible values of $\rho_{S}$. Similar results are obtained by changing $z$ from 0.2 to 0.4 or 0.6.

$p p$ Drell-Yan processes can vary by a factor of up to 2 , depending on which sets of parameters one uses. Mathematically, we have kept the validity of Eq. (63) by letting both $\mathcal{F}_{\text {DIS }}^{S}$ and $A_{\text {DIS }}^{S}$ change, but in opposite ways (if one decreases, the other increases, and vice versa).

We now extend our investigation of what happens to the estimates for the full DY Sivers asymmetry if we let the SIDIS parameters vary in different ways in the $\left(\rho_{S}, \xi_{1}\right)$ space. We first wonder whether it is possible to keep the validity of Eq. (63) by requiring that both $\mathcal{F}_{\text {DIS }}^{S}$ and $A_{\text {DIS }}^{S}$ do not change when moving along some lines in the parameter space [notice that if $A_{\mathrm{DIS}}^{S}$ does not change, then by Eq. (65), $A_{\mathrm{DY}}^{S}$ also does not change]. From Eq. (67), we see that this request amounts to imposing

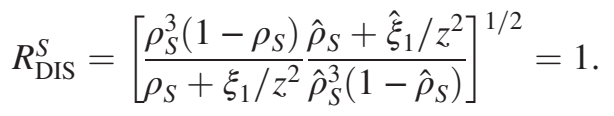

At fixed $\xi_{1}$ and $z$, this constraint corresponds to a fourthorder algebraic equation in the variable $\rho_{S}$ :

$$
\begin{gathered}
\rho_{S}^{4}-\rho_{S}^{3}+\hat{a}(z) \rho_{S}+\hat{a}(z) \frac{z^{2}}{\xi_{1}}=0, \\
\text { with } \hat{a}(z)=\frac{\hat{\rho}_{S}^{3}\left(1-\hat{\rho}_{S}\right)}{\hat{\rho}_{S}+\hat{\xi}_{1} / z^{2}},
\end{gathered}
$$

and we can look for its (real) solutions in terms of $\xi_{1}$ and $z$ in the physical range $0<\rho_{S}<1$. There are in fact two real solutions, at least for some ranges of $\xi_{1}$ values. As an example, they are shown as a function of $\xi_{1}$ and at fixed $z=0.2$ in Fig. 1 for the FIT09 (left panel) and the FIT16 (right panel) cases. The black dots correspond to the position in the parameter plane of the corresponding reference fit. They both belong to the lower of the two possible branches of solutions (the red solid and blue longdashed curves). The corresponding values of $R_{\mathrm{DY}}^{N}\left(=R_{\mathrm{DY}}^{S}\right.$ in this scenario) - that is, the rescaling factor for the predictions of the DY Sivers asymmetry in Eq. (70)-are shown as a function of $\xi_{1}$ in Fig. 2.

The left panel of Fig. 2 shows that the rescaling factor for the set FIT09, for which $\hat{\xi}_{1}^{(09)}=0.80$, decreases to almost $1 / 2$ when $\xi_{1}$ approaches 0.20 , as seen in the previous scenario (notice, however, that in this case $\rho_{S}$ also changes). Concerning the set FIT16 (right panel of Fig. 2), we see that, although the range of $\xi_{1}$ values leading to an allowed value of $\rho_{S}$ is more restricted, in any case the depletion effect on the total DY asymmetry can still be large as soon as $\xi_{1}$ decreases.

Notice that, even if our calculation leads to two possible solutions for $\rho_{S}$ at fixed $\xi_{1}$ (the reference fits corresponding to the lower one), the rescaling factor $R_{\mathrm{DY}}^{N}$, which is the quantity of interest from the physical point of view, is very similar for the two cases. Qualitatively similar results and conclusions apply when considering $z=0.4$ and 0.6.

The plots in Figs. 1 and 2 are shown for all values of $\xi_{1}$ mathematically compatible with the physical request $0<\rho_{S}<1$, but one should not forget that very small values of $\xi_{1}$ are not realistic. Actually, the range $0.15 \lesssim$ $\xi_{1} \lesssim 2.5$ would largely cover most of the parametrizations proposed in the literature (see also Ref. [31]).

Let us finally stress once more that, as compared to the previous scenario, in this case it is the $q_{T}$-integrated component of the overall DY asymmetry that is rescaled by a factor $R_{\mathrm{DY}}^{N}=R_{\mathrm{DY}}^{S}$, while the collinear component is approximately unchanged, since $R_{\mathrm{DIS}}^{S}=1$, see Eqs. (68) and (69).

\section{Sivers effect, scenario 3: Fixing $A_{N}^{\text {DY }}$}

Finally, we wonder whether it is possible to change the parameters $\rho_{S}$ and $\xi_{1}$, moving away from the reference fit values in the parameter space, still getting the same results not only for the $P_{T}$-integrated Sivers SIDIS asymmetry, 

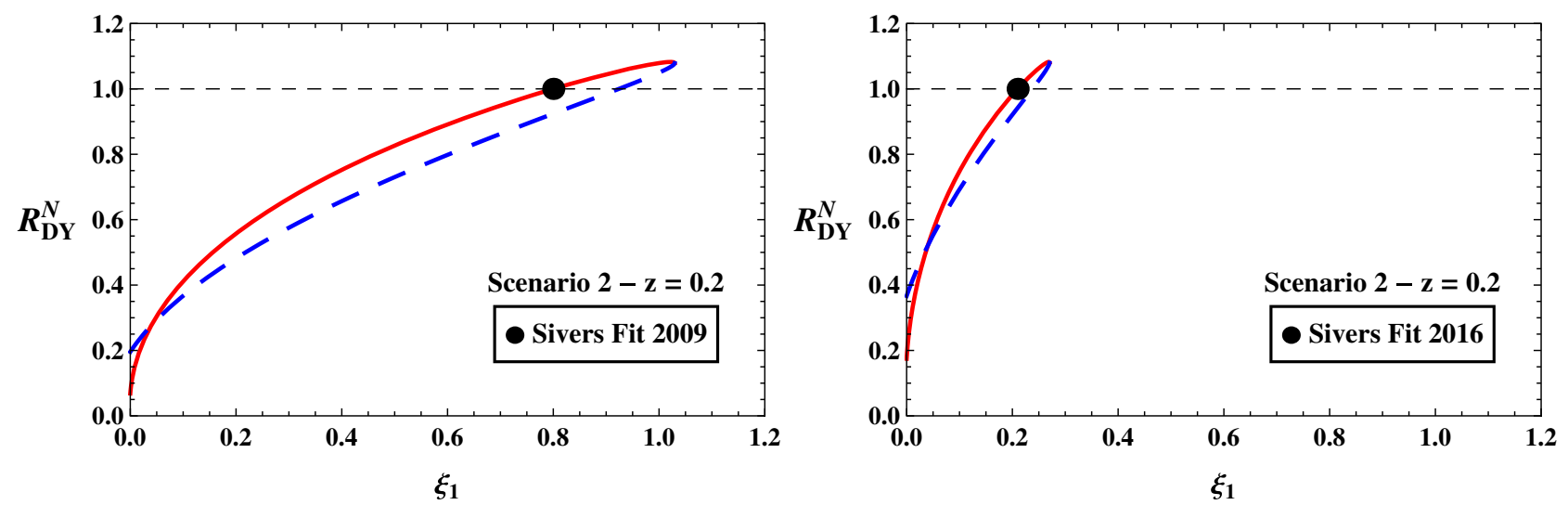

FIG. 2. The plots show how the predictions for the Drell-Yan $q_{T}$-integrated Sivers asymmetry $A_{N}^{\mathrm{DY}}$, see Eq. (55), change, as functions of $\xi_{1}$, when the parameters extracted from SIDIS data move along the corresponding lines of Fig. 1 . The rescaling factor $R_{\mathrm{DY}}^{N}$ is defined in Eqs. (69) and (70). In this scenario the $P_{T}$-integrated SIDIS Sivers asymmetry $A_{U T}^{\sin \left(\phi_{h}-\phi_{S}\right)}$, see Eq. (51), does not change, together with its factors $A_{\text {DIS }}^{S}$ and $\mathcal{F}_{\text {DIS. }}^{S}$.

$A_{U T}^{\sin \left(\phi_{h}-\phi_{S}\right)}$, but also for the $q_{T}$-integrated Sivers DY asymmetry, $A_{N}^{\mathrm{DY}}$. This amounts to requesting that

$$
R_{\mathrm{DY}}^{N}=\left[\frac{\rho_{S}+\xi_{1} / z^{2}}{\hat{\rho}_{S}+\hat{\rho}_{S}+z^{2}} \frac{1}{\rho_{S}+1}\right]^{1 / 2}=1,
$$

or, equivalently,

$$
R_{\mathrm{DY}}^{S}=R_{\mathrm{DIS}}^{S}
$$

By defining

$$
\hat{b}(z)=\frac{\hat{\rho}_{S}+1}{\hat{\rho}_{S}+\hat{\xi}_{1} / z^{2}},
$$

Equation (75) translates into the simple linear relation

$$
\rho_{S}=\frac{\hat{b}(z) \xi_{1} / z^{2}-1}{1-\hat{b}(z)} \quad \text { for } \hat{b}(z) \neq 1,
$$

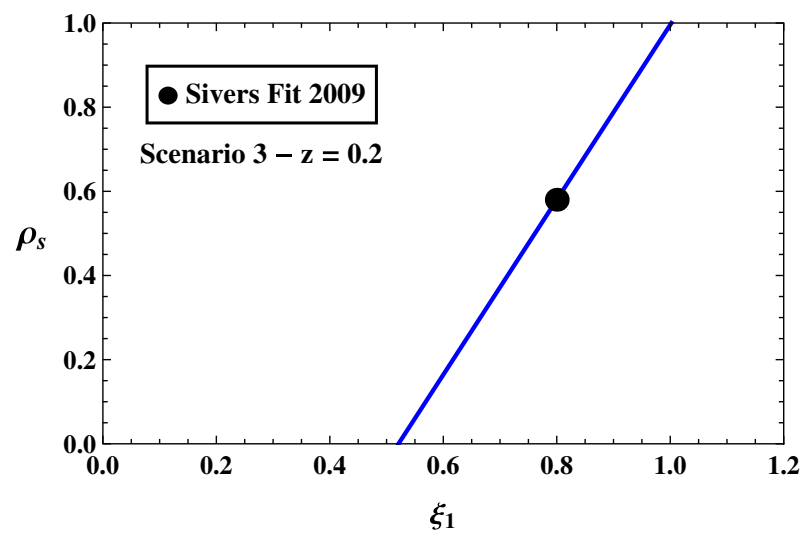

where $\hat{b}(z)$ is a rapidly increasing function of $z$. To give an idea, $\quad \hat{b}^{(09)}(z=0.2) \simeq 0.08, \quad \hat{b}^{(09)}(z=0.6) \simeq 0.56$, $\hat{b}^{(16)}(z=0.2) \simeq 0.27, \quad \hat{b}^{(16)}(z=0.6) \simeq 1.36$. Requiring that $0<\rho_{S}<1$ restricts the allowed values of $\xi_{1}$ in terms of $\hat{b}(z)$ :

$$
\begin{aligned}
& \text { if } \hat{b}<1, \quad \text { then } z^{2} / \hat{b}<\xi_{1}<z^{2}(2-\hat{b}) / \hat{b}, \\
& \text { if } \hat{b}>1, \quad \text { then } z^{2}(2-\hat{b}) / \hat{b}<\xi_{1}<z^{2} / \hat{b} .
\end{aligned}
$$

As an example, Fig. 3 shows, for the sets FIT09 (left panel) and FIT16 (right panel) and for $z=0.2$, the values of $\rho_{S}$ corresponding to the allowed $\xi_{1}$ range that keep fixed the values of both the total Drell-Yan and SIDIS Sivers asymmetries when moving away from the $\left(\hat{\rho}_{S}, \hat{\xi}_{1}\right)$ values of the corresponding fit.

The slope of the straight lines in the plots increases, and therefore the allowed range for $\xi_{1}$ shrinks, as $\hat{b}(z)$

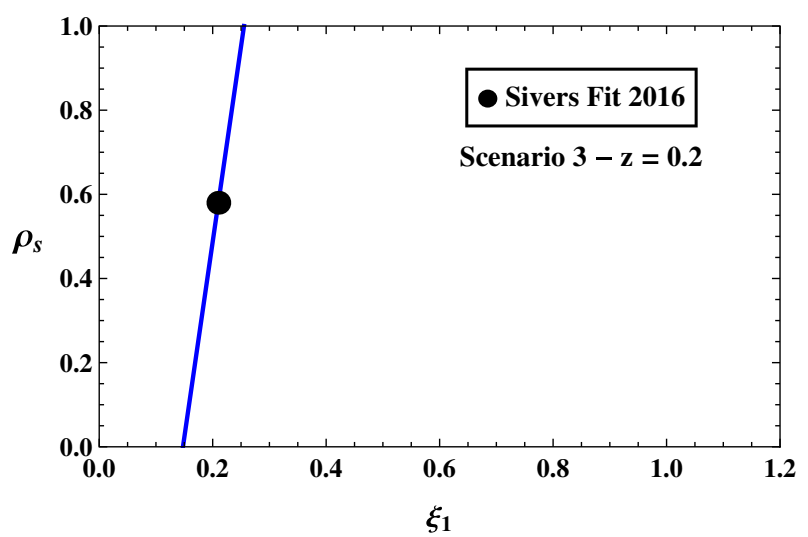

FIG. 3. The two lines in the $\left(\rho_{S}, \xi_{1}\right)$ parameter space show the set of values of $\rho_{S}$ and $\xi_{1}$ which leave unchanged the SIDIS Sivers asymmetry $A_{U T}^{\sin \left(\phi_{h}-\phi_{S}\right)}$, see Eq. (51), and the predictions for the Drell-Yan Sivers asymmetry $A_{N}^{\mathrm{DY}}$, see Eq. (69). The black dots correspond to the values $\hat{\rho}_{S}$ and $\hat{\xi}_{1}$ obtained in the fits of Ref. [17] (left plot, FIT09) and of Ref. [23] (right plot, FIT16), which describe equally well the SIDIS Sivers asymmetry. 

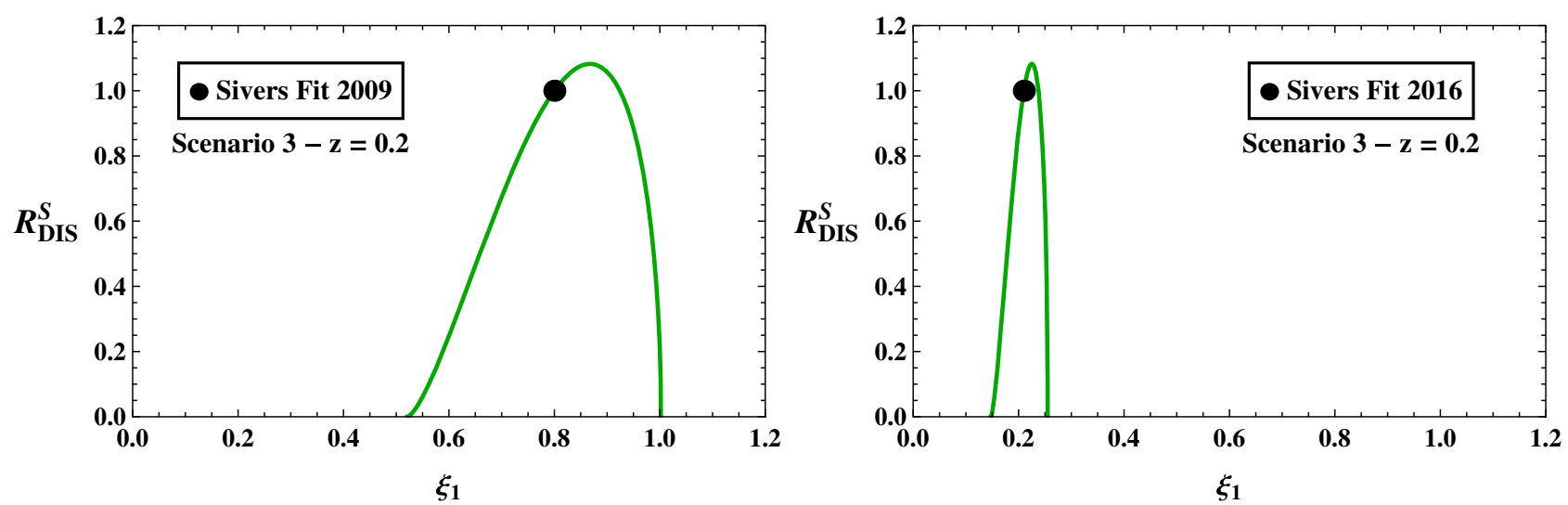

FIG. 4. The plots show the rescaling factor $R_{\mathrm{DIS}}^{S}=R_{\mathrm{DY}}^{S}$ which fixes the changes of $\mathcal{F}_{\mathrm{DIS}}^{S}$ and $\mathcal{F}_{\mathrm{DY}}^{S}$, see Eqs. (67) and (68), when the parameters $\rho_{S}$ and $\xi_{1}$ move as in the corresponding plots of Fig. 3. Notice that $A_{\mathrm{DIS}}^{S}$ and $A_{\mathrm{DY}}^{S}$, and therefore the collinear component of the Sivers function, change as $1 / R_{\mathrm{DIS}}^{S}$. In this scenario, both the $P_{T}$-integrated SIDIS Sivers asymmetry $A_{U T}^{\sin \left(\phi_{h}-\phi_{S}\right)}$, see Eq. $(51)$, and the $q_{T}$-integrated DY Sivers asymmetry $A_{N}^{\mathrm{DY}}$, see Eq. (55), do not change.

approaches 1 , changing sign when it crosses this value. For $\hat{b}(z)=1$ - that is, for $0<z=\hat{\xi}_{1}^{1 / 2}<1$-Eq. (75) can be fulfilled only for $\xi_{1} \equiv \hat{\xi}_{1}$, and $\rho_{S}$ is undetermined.

Notice that in this case, like in scenario 1, although the total Sivers asymmetries are unchanged, the separate factors depending on the longitudinal momentum fractions and on the transverse momenta, $A^{S}$ and $\mathcal{F}^{S}$ respectively, change according to Eqs. (67), (68), and (76). The rescaling factor $R_{\mathrm{DIS}}^{S}=R_{\mathrm{DY}}^{S}$ is shown in Fig. 4 as a function of $\xi_{1}$ in the allowed range, for the two reference fits.

By comparing Figs. 1 and 3, we see that future $q_{T}$-integrated data on the Drell-Yan Sivers asymmetries could constrain more severely the range of allowed $\xi_{1}$ values-in particular, for the FIT16 set (right panel of Fig. 3). On the other hand, Fig. 4 shows that even in this restricted range, as soon as the value of $\xi_{1}$ changes (with respect to that of the reference fits), the two factors of the total asymmetry $-A^{S}$, which depends on the longitudinal momentum fractions and $\mathcal{F}^{S}$, which depends on the transverse momenta—can change by a sizeable factor. For $A^{S}$, this implies a sizeable change in the collinear part of the Sivers distribution function $\Delta^{N} f_{q / p^{\uparrow}}(x)$, like $1 / R_{\text {DIS }}^{S}$.

Again, the plots shown in Fig. 4 cover all variable ranges mathematically allowed, but one should keep in mind that too small values of $R_{\mathrm{DIS}}^{S}$ are not physically acceptable. Such values would yield large values of $A_{\mathrm{DIS}}^{S}$ and $A_{\mathrm{DY}}^{S}$ [see Eqs. (67) and (68)], and consequently, large values of $\Delta f_{q / p^{\uparrow}}(x)$, which eventually violate the positivity bound $\left|\Delta f_{q / p^{\uparrow}}(x)\right| \leq 2 f_{q / p}(x)$.

These results, and those of the previous two scenarios, clearly show how the choice of a specific set for the Gaussian widths of the unpolarized TMDs could play a crucial role in the extraction of the Sivers function from the analysis of the corresponding SIDIS azimuthal asymmetries and, as a consequence, in the predictions for the Sivers asymmetries in DY processes.

\section{THE COLLINS CASE}

Let us now extend the considerations of the previous section to the Collins asymmetries and see how the uncertainty on the choice of $\xi_{1}$ can affect the extraction of the transversity distribution and the Collins function from SIDIS and $e^{+} e^{-}$annihilation data, see Eqs. (53), (54) and (57), (58). Notice that although $\mathcal{F}_{\text {ee }}^{C}\left(\rho_{C}\right)$ does not depend explicitly on $\xi_{1}$, possible conditions on $\mathcal{F}_{\text {DIS }}^{C}$ induce a correlation between $\rho_{C}$ and $\xi_{1}$.

The Collins case is more complicated than the Sivers case. In fact, in the latter case the explored Sivers function always enters linearly, convoluted either with the unpolarized FF function in the SIDIS asymmetries, or with the unpolarized PDF in the DY asymmetries. Instead, in the Collins asymmetries, the Collins FF enters linearly in the SIDIS casecoupled to the transversity distribution-while in the $e^{+} e^{-}$ case it appears "quadratically," in the sense that the Collins function associated with hadron $h_{1}$ is convoluted with the Collins function associated with hadron $h_{2}$. This makes the analysis less direct, since variations in the transversemomentum-dependent factors can generate different effects on the $x$ - and $z$-dependent parts. More precisely, they can affect only the transversity distribution $h_{1}^{q}(x)$, or only the collinear part of the Collins FF $\Delta^{N} D_{h / q^{\uparrow}}(z)$, or both of them simultaneously.

Moreover, no experimental data are presently available on the $p_{\perp}$ distributions in the cross section for $e^{+} e^{-} \rightarrow$ $h_{1} h_{2} X$ processes, from which one could attempt an extraction of the unpolarized $\left\langle p_{\perp}^{2}\right\rangle$ width. Old measurements exist, that were recently analyzed in Ref. [44], but they correspond to single hadron production in $e^{+} e^{-}$ annihilations, a process for which TMD factorization theorems are not proven to be applicable.

In order to simplify our discussion, we assume that changes in the values of $\xi_{1}$ and $\rho_{C}$ will possibly be reflected 
only in variations of the overall numerical factors appearing in the collinear parts of the transversity distribution and the Collins FF, rather than in their functional shapes. At the qualitative level of the present treatment, this allows us to focus on the main effects, avoiding additional complications. For the same reason, we take $\xi_{T}=\left\langle k_{\perp}^{2}\right\rangle_{T} /\left\langle k_{\perp}^{2}\right\rangle=1$, assuming that the transversity distribution has the same transverse momentum dependence as the unpolarized TMDs.

As for the Sivers case, in our analysis we consider two different reference parametrizations for the transversity distribution and the Collins FF with comparable accuracies of the corresponding fits to data:

(1) The fit of Ref. [24], referred to as FIT07 in the following, for which

$$
\begin{aligned}
\left\langle k_{\perp}^{2}\right\rangle & =0.25 \mathrm{GeV}^{2}, \quad\left\langle p_{\perp}^{2}\right\rangle=0.20 \mathrm{GeV}^{2}, \\
M_{C}^{2} & =0.88 \mathrm{GeV}^{2},
\end{aligned}
$$

implying

$$
\hat{\xi}_{1}^{(07)}=0.80, \quad \hat{\rho}_{C}^{(07)}=0.81 .
$$

The complete list of parameters can be found in Table II of Ref. [24], where more details on the fitting procedure, the parameter extraction, and additional references are given.

(2) The fit from Ref. [21], referred to as FIT15, for which

$$
\begin{aligned}
\left\langle k_{\perp}^{2}\right\rangle & =0.57 \mathrm{GeV}^{2}, \quad\left\langle p_{\perp}^{2}\right\rangle=0.12 \mathrm{GeV}^{2}, \\
M_{C}^{2} & =0.28 \mathrm{GeV}^{2},
\end{aligned}
$$

corresponding to

$$
\hat{\xi}_{1}^{(15)}=0.21, \quad \hat{\rho}_{C}^{(15)}=0.70 .
$$

Again, full details and the complete list of parameters can be found in Ref. [21] and its Table I.

We recall that for the Collins asymmetry in $e^{+} e^{-}$ annihilations we have considered here the $A_{0}$ asymmetry, corresponding to the experimental "hadronic plane" setup, where no direct reference to the $q \bar{q}$ jet thrust axis is made (see Ref. [21] and references therein). Notice also that, similarly to the SIDIS Sivers case, the two reference fits differ significantly in the values of $\hat{\xi}_{1}$ and much less in the values of $\hat{\rho}_{C}$ ( $\hat{\rho}_{S}$ for the Sivers asymmetry).

In the Sivers case discussed in the previous section, we investigated how the freedom left on the parameters $\xi_{1}$ and $\rho_{S}$ by SIDIS data could affect the predictions for the Sivers asymmetry in DY processes. This was because of the lack of experimental information on polarized DY scattering experiments. In the case of the Collins asymmetry, instead, sufficient experimental information is available both from
SIDIS and $e^{+} e^{-}$annihilation data. We then investigate the freedom left on the parameters $\xi_{1}$ and $\rho_{C}$ by these data; that is, we study whether, moving in the parameter space $\left(\rho_{C}\right.$, $\left.\xi_{1}\right)$ away from a given reference set $\left(\hat{\rho}_{C}, \hat{\xi}_{1}\right)$, the following relations remain true:

$$
\begin{aligned}
A_{\mathrm{DIS}}^{C} \mathcal{F}_{\mathrm{DIS}}^{C}\left(\rho_{C}, \xi_{1}\right) & \simeq \hat{A}_{\mathrm{DIS}}^{C} \hat{\mathcal{F}}_{\mathrm{DIS}}^{C}\left(\hat{\rho}_{C}, \hat{\xi}_{1}\right), \\
A_{\mathrm{ee}}^{C} \mathcal{F}_{\mathrm{ee}}^{C}\left(\rho_{C}\right) & \simeq \hat{A}_{\mathrm{ee}}^{C} \hat{\mathcal{F}}_{\mathrm{ee}}^{C}\left(\hat{\rho}_{C}\right) .
\end{aligned}
$$

Notice that by using Eq. (54), with $\xi_{T}=1$, and Eq. (58), in complete analogy with the Sivers case, we can also write

$$
\mathcal{F}_{\text {DIS }}^{C}=R_{\text {DIS }}^{C} \hat{\mathcal{F}}_{\text {DIS }}^{C}, \quad A_{\text {DIS }}^{C} \simeq \frac{1}{R_{\text {DIS }}^{C}} \hat{A}_{\text {DIS }}^{C},
$$

with $\quad R_{\mathrm{DIS}}^{C}=\left[\frac{\rho_{C}^{3}\left(1-\rho_{C}\right)}{\rho_{C}+z^{2} / \xi_{1}} \frac{\hat{\rho}_{C}+z^{2} / \hat{\xi}_{1}}{\hat{\rho}_{C}^{3}\left(1-\hat{\rho}_{C}\right)}\right]^{1 / 2}$

and

$$
\begin{aligned}
\mathcal{F}_{\mathrm{ee}}^{C} & =R_{\mathrm{ee}}^{C} \hat{\mathcal{F}}_{\mathrm{ee}}^{C}, \quad A_{\mathrm{ee}}^{C} \simeq \frac{1}{R_{\mathrm{ee}}^{C}} \hat{A}_{\mathrm{ee}}^{C}, \\
\text { with } \quad R_{\mathrm{ee}}^{C} & =\frac{\rho_{C}^{2}\left(1-\rho_{C}\right)}{\hat{\rho}_{C}^{2}\left(1-\hat{\rho}_{C}\right)} .
\end{aligned}
$$

Due to the factorized nature of our approach, there could be several solutions of Eq. (84). We consider as examples a few possible scenarios which differ by one further additional condition, leading to different ways of modifying the collinear and transverse-momentum-dependent terms in the asymmetries, and ultimately, the corresponding components of the transversity distribution $h_{1}^{q}(x)$ and of the Collins FF $\Delta^{N} D_{h / q^{\uparrow}}(z)$.

\section{A. Collins effect, scenario 1}

In this scenario, we look for possible allowed sets of $\left(\rho_{C}, \xi_{1}\right)$ values which not only leave unchanged the two (SIDIS and $e^{+} e^{-}$) Collins asymmetries in Eq. (84), but also the $P_{T}$-integrated SIDIS Collins factor $\mathcal{F}_{\text {DIS }}^{C}$ :

$$
\mathcal{F}_{\text {DIS }}^{C}\left(\rho_{C}, \xi_{1}\right)=\hat{\mathcal{F}}_{\text {DIS }}^{C}\left(\hat{\rho}_{C}, \hat{\xi}_{1}\right),
$$

that is,

$$
R_{\mathrm{DIS}}^{C}=\left[\frac{\rho_{C}^{3}\left(1-\rho_{C}\right)}{\rho_{C}+z^{2} / \xi_{1}} \hat{\rho}_{C}+z^{2} / \hat{\xi}_{1}^{3}\left(1-\hat{\rho}_{C}\right)\right]^{1 / 2}=1 .
$$

As for the Sivers case, the above constraint corresponds to a fourth-order algebraic equation for $\rho_{C}$, at fixed $\hat{\xi}_{1}, \hat{\rho}_{C}$, and $z$ :

$$
\begin{gathered}
\rho_{C}^{4}-\rho_{C}^{3}+\hat{c}(z) \rho_{C}+\hat{c}(z) \frac{z^{2}}{\xi_{1}}=0, \\
\text { with } \hat{c}(z)=\frac{\hat{\rho}_{C}^{3}\left(1-\hat{\rho}_{C}\right)}{\hat{\rho}_{C}+z^{2} / \hat{\xi}_{1}} .
\end{gathered}
$$



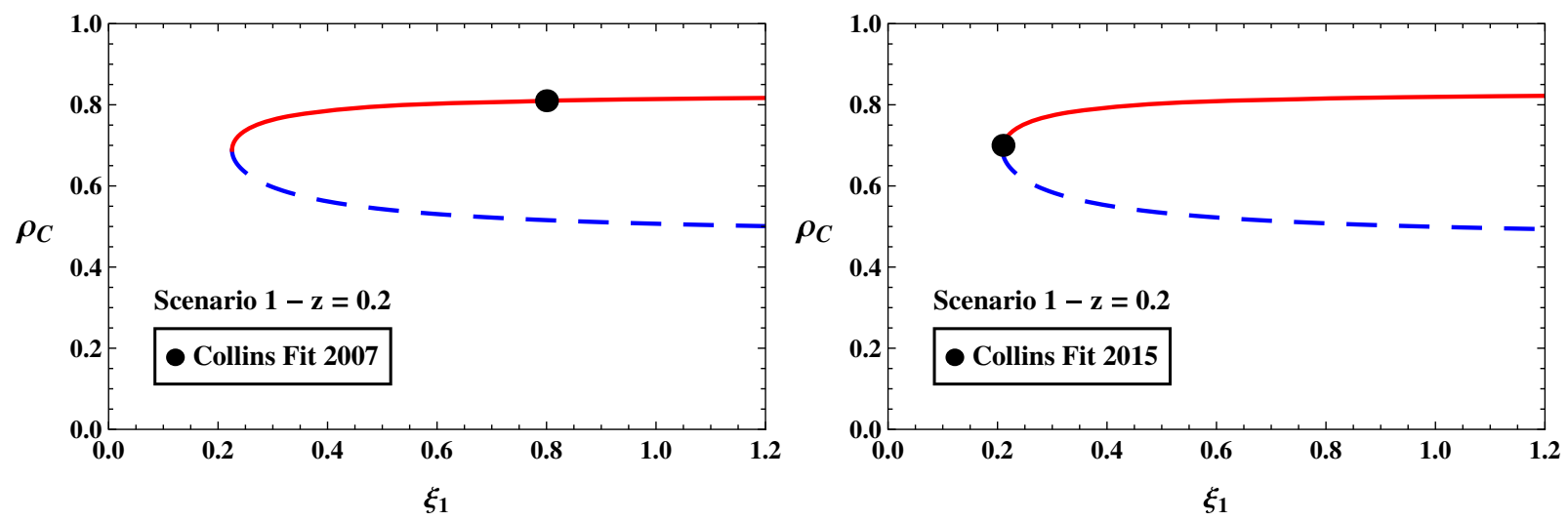

FIG. 5. The curves in the $\left(\rho_{C}, \xi_{1}\right)$ parameter space show the set of values of $\rho_{C}$ and $\xi_{1}$ which leave unchanged the $P_{T}$-integrated factor of the Collins asymmetry, $\mathcal{F}_{\text {DIS }}^{C}(z=0.2)$. The black dots correspond to the values $\hat{\rho}_{C}$ and $\hat{\xi}_{1}$ obtained in the fits of Ref. [24] (left plot, FIT07) and of Ref. [21] (right plot, FIT15), which describe equally well the SIDIS and $e^{+} e^{-}$Collins asymmetries. Notice that for each value of $\xi_{1}$, one finds two possible values of $\rho_{C}$. Similar results are obtained by changing $z$ from 0.2 to 0.4 or 0.6 .

Again, it turns out that two of the four possible solutions for $\rho_{C}$ are complex, while the other two can be real, at least for some range of $\xi_{1}$ values. As an example, they are shown in Fig. 5, for both the FIT07 (left panel) and FIT15 (right panel) parametrizations, as a function of $\xi_{1}$ at fixed $z=0.2$. Notice that, although the plots are shown up to $\xi_{1}=1.2$, at variance with $\rho_{S}$ for the Sivers case, the two solutions for $\rho_{C}$ survive, almost constant, up to much larger $\xi_{1}$ values.

From Eqs. (85) and (86) we have, in this scenario,

$$
A_{\mathrm{DIS}}^{C}=\hat{A}_{\mathrm{DIS}}^{C}, \quad A_{\mathrm{ee}}^{C}=\frac{1}{R_{\mathrm{ee}}^{C}} \hat{A}_{\mathrm{ee}}^{C} .
$$

Let us recall that $A_{\text {DIS }}^{C}$ in Eq. (24) is a linear convolution of the transversity distribution $h_{1}^{q}(x)$ and the collinear component of the Collins function $\Delta^{N} D_{h / q^{\uparrow}}(z)$, while $A_{\text {ee }}^{C}$ in Eq. (47) is "quadratic" in $\Delta^{N} D_{h / q^{\uparrow}}$. Then, it is reasonable to assume that, in order to keep satisfying
Eq. (90) while the parameters $\left(\rho_{c}, \xi_{1}\right)$ vary as in Fig. 5, $\Delta^{N} D_{h / q^{\uparrow}}$ rescales, approximately, as $1 / \sqrt{R_{\text {ee }}^{C}}$ and, as a consequence, $h_{1}^{q}(x)$ must rescale as $\sqrt{R_{\mathrm{ee}}^{C}}$. This rescaling factor is shown in Fig. 6 for each of the two possible solutions $\rho_{C}\left(\xi_{1}\right)$.

This figure shows that in the range of $\xi_{1}$ considered, the rescaling factor differs from unity by a factor of $\pm 10 \%$ at most, which is well inside the uncertainties of the extraction procedure [21,24]. However, as $z$ increases to 0.6 , the allowed range of $\xi_{1}$ shrinks to larger values for the FIT07 case, while for the FIT15 set the rescaling factor $\sqrt{R_{\mathrm{ee}}^{C}}$ decreases to 0.6 at larger $\xi_{1}$.

Let us also notice that in this scenario, and within a phenomenological TMD approach, the possible Collins contribution to SSAs in $p^{\uparrow} p \rightarrow h X, p^{\uparrow} p \rightarrow h$ jet $X$ processes should remain approximately unchanged, like in the SIDIS case, since the transversity distribution and the
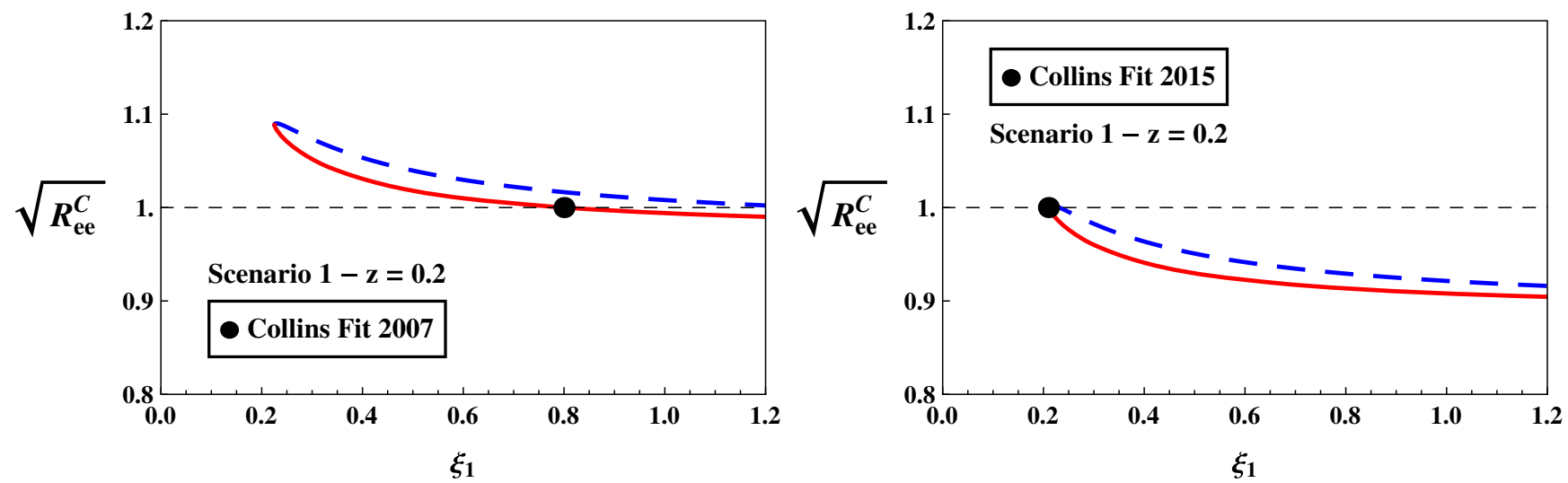

FIG. 6. The expected rescaling factor $\sqrt{R_{\mathrm{ee}}^{C}}$ for the collinear transversity distribution $h_{1}^{q}(x)$ when the parameters $\rho_{C}$, $\xi_{1}$ move away from the reference fit values as in the corresponding plots of Fig. 5. Simultaneously, the Collins collinear distribution $\Delta^{N} D_{h / q^{\uparrow}}(z)$ rescales as $1 / \sqrt{R_{\mathrm{ee}}^{C}}$. In this scenario the total $P_{T^{-}}$-integrated Collins asymmetries, Eqs. (55) and (57), remain unchanged, as well as the $\mathcal{F}_{\text {DIS }}^{C}$ factor. 

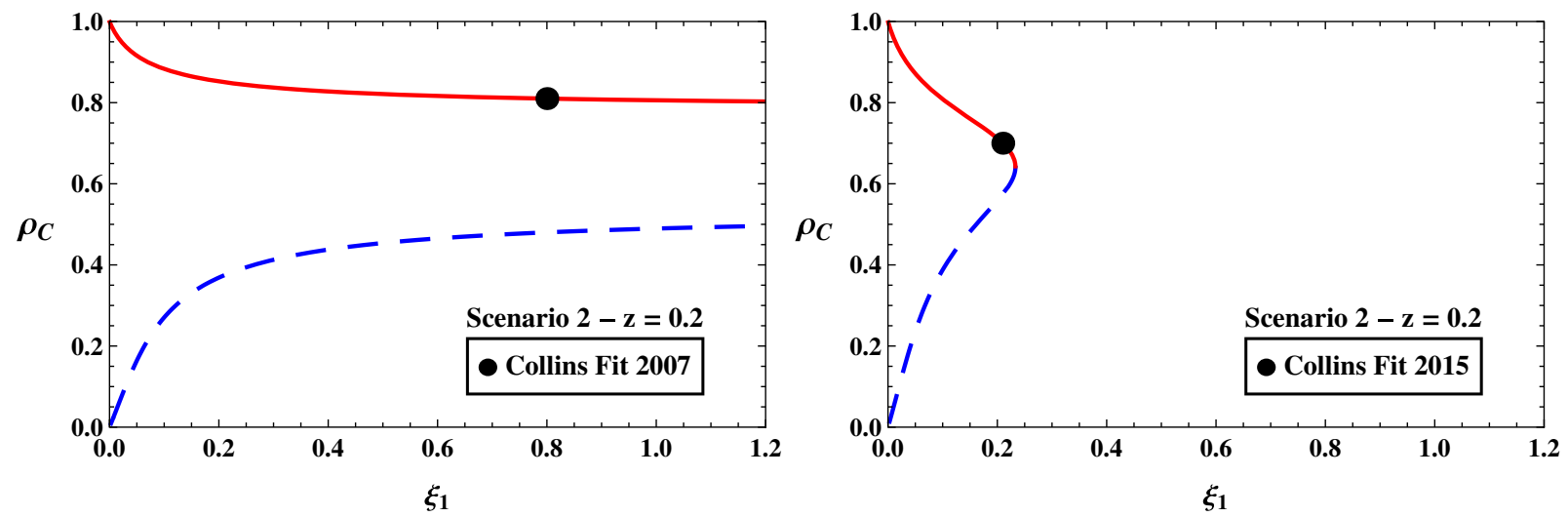

FIG. 7. The curves in the $\left(\rho_{C}, \xi_{1}\right)$ parameter space show the set of values of $\rho_{C}$ and $\xi_{1}$ which satisfy Eq. (92) at $z=0.2$. The black dots correspond to the values $\hat{\rho}_{C}$ and $\hat{\xi}_{1}$ obtained in the fits of Ref. [24] (left plot, FIT07) and of Ref. [21] (right plot, FIT15), which describe equally well the SIDIS and $e^{+} e^{-}$Collins asymmetries. Notice that for each value of $\xi_{1}$, one finds two possible values of $\rho_{C}$. Similar results are obtained by changing $z$ from 0.2 to 0.4 or 0.6 .

Collins FF change simultaneously by an inverse overall factor.

\section{B. Collins effect, scenario 2}

In this scenario, we still require that the two Collins asymmetries for SIDIS and $e^{+} e^{-}$collisions remain approximately unchanged [Eq. (84)], imposing this time as a further condition that the transverse-momentum-dependent terms of the two Collins asymmetries change in the same way:

$$
\frac{\mathcal{F}_{\text {ee }}^{C}}{\hat{\mathcal{F}}_{\text {ee }}^{C}}=\frac{\mathcal{F}_{\text {DIS }}^{C}}{\hat{\mathcal{F}}_{\text {DIS }}^{C}},
$$

that is, from Eqs. (85) and (86),

$$
R_{\mathrm{DIS}}^{C}=R_{\mathrm{ee}}^{C}
$$

At fixed $\hat{\xi}_{1}, \hat{\rho}_{C}, z$, the above constraint translates into an algebraic cubic equation for $\rho_{C}\left(\xi_{1}\right)$ :

$$
\begin{aligned}
& \rho_{C}^{3}+\left(\frac{z^{2}}{\xi_{1}}-1\right) \rho_{C}^{2}-\frac{z^{2}}{\xi_{1}} \rho_{C}+\hat{d}(z)=0 \\
& \text { with } \hat{d}(z)=\hat{\rho}_{C}\left(1-\hat{\rho}_{C}\right)\left(\hat{\rho}_{C}+\frac{z^{2}}{\hat{\xi}_{1}}\right) .
\end{aligned}
$$

Only two out of the three solutions are real in the range of $\xi_{1}$ values of interest. As an illustration, they are shown in Fig. 7 for both the FIT07 (left panel) and FIT15 (right panel) parametrizations, as a function of $\xi_{1}$ at fixed $z=0.2$. Notice that the solutions for the FIT07 case, shown on the left panel, survive, almost constant, up to values of $\xi_{1}$ much larger than those shown in the plot.
The condition (92) implies

$$
\frac{A_{\mathrm{ee}}^{C}}{\hat{A}_{\mathrm{ee}}^{C}}=\frac{A_{\mathrm{DIS}}^{C}}{\hat{A}_{\mathrm{DIS}}^{C}}=\frac{1}{R_{\mathrm{DIS}}^{C}}=\frac{1}{R_{\mathrm{ee}}^{C}},
$$

which corresponds to a situation in which both the collinear terms of the transversity distribution, $h_{1}^{q}(x)$, and that of the Collins fragmentation function, $\Delta^{N} D_{h / q^{\uparrow}}(z)$, are approximately rescaled by the same factor $1 / \sqrt{R_{\text {DIS }}^{C}}$.

For each of the two possible solutions $\rho_{C}\left(\xi_{1}\right)$ shown in Fig. 7 , the corresponding rescaling factor $1 / \sqrt{R_{\text {DIS }}^{C}}=$ $1 / \sqrt{R_{\mathrm{ee}}^{C}}$ is shown in Fig. 8, as a function of $\xi_{1}$ at fixed $z=0.2$, for the FIT07 (left panel) and the FIT15 (right panel) cases. For the FIT07 case, we see that, with the exception of the very small $\xi_{1}$ region, the rescaling factor is not far from unity, for both branches of $\rho\left(\xi_{1}\right)$. For the FIT15 case, the rescaling factor can be remarkably different for the two solutions, and for one of them can be sizeably larger than unity; however, as we commented before, the region $\xi_{1} \lesssim 0.2$ is unlikely to be a physical one.

As $z$ increases to 0.6 , for the FIT07 case the rescaling factor for the two solutions differs more and can reach values sensibly different from unity already for not very small $\xi_{1}$, of the order $0.4 \div 0.5$.

\section{Collins effect, scenario 3}

Finally, we consider a scenario based on Eq. (84) and the further constraint that the collinear and the $q_{T}$-integrated components of the Collins asymmetry for $e^{+} e^{-}$annihilations remain separately fixed. According to Eq. (86), this corresponds to the condition

$$
R_{\mathrm{ee}}^{C}=1,
$$

that is,

$$
\rho_{C}^{3}-\rho_{C}^{2}+\hat{\rho}_{C}^{2}\left(1-\hat{\rho}_{C}\right)=0 .
$$



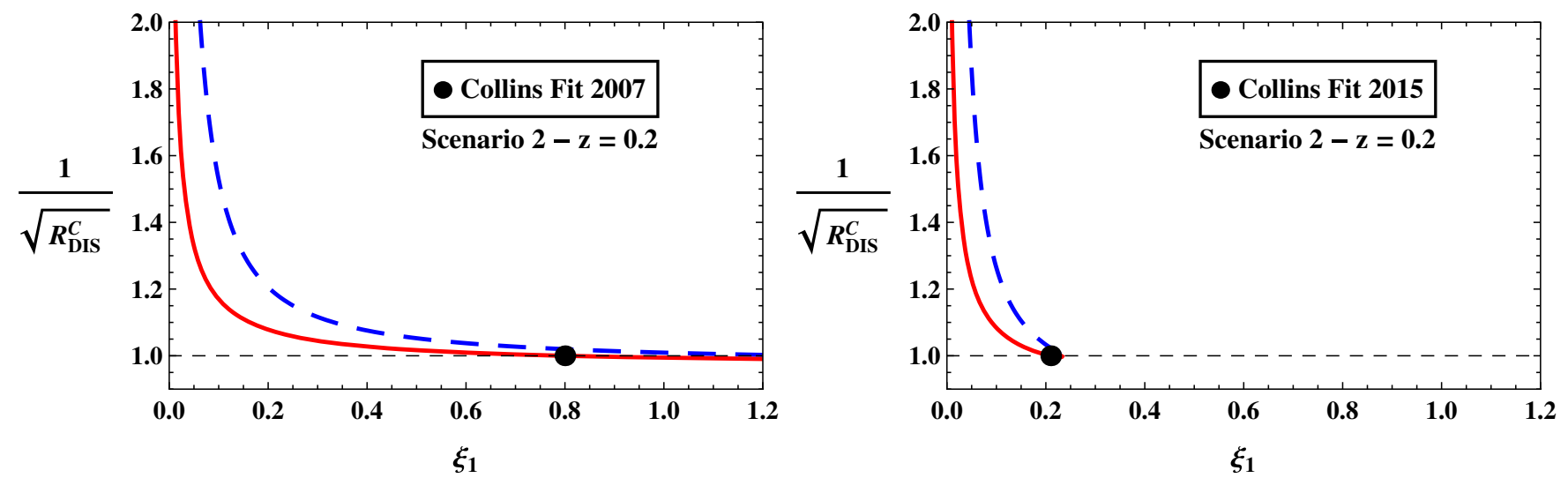

FIG. 8. The expected rescaling factor $1 / \sqrt{R_{\mathrm{DIS}}^{C}}$ for the collinear transversity distribution $h_{1}^{q}(x)$ and the collinear Collins function $\Delta^{N} D_{h / q^{\uparrow}}(z)$ when the parameters $\rho_{C}, \xi_{1}$ move away from the reference fit values as in the corresponding plots of Fig. 7. In this scenario, the total $P_{T}$-integrated Collins asymmetries, Eqs. (55) and (57), remain unchanged, while $\mathcal{F}_{\text {DIS }}^{C}$ and $\mathcal{F}_{\text {ee }}^{C}$ rescale in the same way.
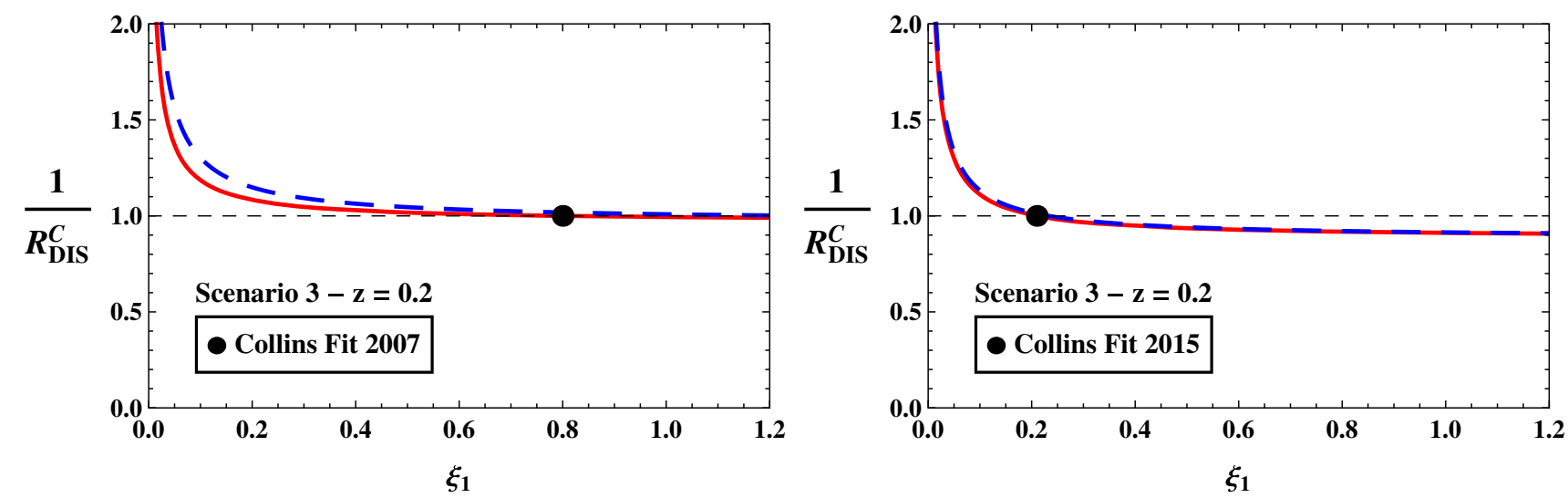

FIG. 9. The expected rescaling factor $1 / R_{\text {DIS }}^{C}$ for the collinear transversity distribution $h_{1}^{q}(x)$, as a function of $\xi_{1}$ at fixed $z=0.2$, when the parameter $\rho_{C}$ assumes the values given in Eq. (98) (left plot) and in Eq. (99) (right plot). In this scenario, the total $P_{T}$-integrated Collins asymmetries, Eqs. (55) and (57), remain unchanged, as well as the $\mathcal{F}_{\text {ee }}^{C}$ factor.

This equation has the following analytical solutions:

$$
\begin{aligned}
& \rho_{C}=\hat{\rho}_{C}, \quad \rho_{C}=\frac{1}{2}\left(1-\hat{\rho}_{C}-\sqrt{1+2 \hat{\rho}_{C}-3 \hat{\rho}_{C}^{2}}\right), \\
& \rho_{C}=\frac{1}{2}\left(1-\hat{\rho}_{C}+\sqrt{1+2 \hat{\rho}_{C}-3 \hat{\rho}_{C}^{2}}\right) .
\end{aligned}
$$

The second root is always negative in the physical range $0<\hat{\rho}_{C}<1$, while the other two take the values

$$
\begin{array}{ll}
\rho_{C}^{(07) 1}=\hat{\rho}_{C}^{(07)}=0.81, & \rho_{C}^{(07) 2}=0.50, \\
\rho_{C}^{(15) 1}=\hat{\rho}_{C}^{(15)}=0.70, & \rho_{C}^{(07) 2}=0.63,
\end{array}
$$

respectively, for the FIT07 and FIT15 cases.
From Eqs. (85) and (86) we have, in this scenario,

$$
A_{\mathrm{ee}}^{C}=\hat{A}_{\mathrm{ee}}^{C}, \quad A_{\mathrm{DIS}}^{C}=\frac{1}{R_{\mathrm{DIS}}^{C}} \hat{A}_{\mathrm{DIS}}^{C}
$$

from which one expects a situation in which the collinear component of the Collins FF, $\Delta^{N} D_{h / q^{\uparrow}}(z)$, remains unchanged, while the transversity distribution $h_{1}^{q}(x)$ changes by a factor $1 / R_{\text {DIS }}^{C}$. The behavior of this rescaling factor $1 / R_{\mathrm{DIS}}^{C}\left(\rho_{C}, \xi_{1}, z\right)$ is shown in Fig. 9 as a function of $\xi_{1}$ at fixed $z=0.2$ for the FIT07 (left panel) and the FIT15 (right panel) cases, and in each case for the two allowed $\rho_{C}$ solutions, Eqs. (98) and (99).

One can see that in both cases, the rescaling factor is very similar for the two possible values of $\rho_{C}$ and is almost equal to 1 , apart from the small unphysical $\xi_{1}$ region. As $z$ increases to 0.6 , the difference between the two solutions is more pronounced for the FIT07 case, and the rescaling factor differs more sizeably from unity in both cases. 


\section{CONCLUSIONS}

We have investigated to what extent the actual parametrizations of the most studied TMDs - the Sivers distribution and the Collins fragmentation function - can be fixed by data and what uncertainties could remain. We feel that such a study is necessary at this stage of the exploration of the 3D nucleon structure, just before a full implementation of the TMD evolution is performed and when new amounts of data are soon expected from COMPASS, JLab 12 , and hopefully in the not so far future, from the electron ion collider (EIC).

We have done so motivated by the observation that most data originate from SIDIS processes in which the parton distribution and fragmentation properties both contribute to build up the final observables, like in Eq. (13), which clearly shows a strong correlation between $\left\langle k_{\perp}^{2}\right\rangle$ and $\left\langle p_{\perp}^{2}\right\rangle$. Indeed, equally good fits of SIDIS asymmetry data could be obtained with rather different values of these two parameters. On the other hand, other processes, like lepton pair production in hadronic collisions (DY) or hadron pair production in $e^{+} e^{-}$annihilations, are only sensitive to the TMD parton distributions or the TMD parton fragmentation functions, respectively.

We have assumed a simple scheme, mainly so far adopted, in which the collinear and transverse degrees of freedom of the TMDs are factorized, with Gaussian dependences for the transverse-momentum-dependent components [Eqs. (2)-(4)]. We have limited our considerations to the $P_{T^{-}}$or $q_{T^{-}}$integrated asymmetries [Eqs. (51)-(58)], which have a very simple structure according to which the Gaussian transverse dependence of the TMDs results in factors which are functions of the Gaussian widths. A change in such parameters, like $\left\langle k_{\perp}^{2}\right\rangle$ and $\left\langle p_{\perp}^{2}\right\rangle$, may affect the extraction of the collinear part of the TMDs.

We have considered separately the extraction of the Sivers and the Collins TMDs. The former is related to measured azimuthal asymmetries in polarized SIDIS, and to so far not yet well known asymmetries in polarized DY processes. The latter is related to measured azimuthal asymmetries in polarized SIDIS and in unpolarized $e^{+} e^{-}$ annihilation processes.

We have found that special care must be taken of the uncertainty in the ratio $\xi_{1}=\left\langle p_{\perp}^{2}\right\rangle /\left\langle k_{\perp}^{2}\right\rangle$ when discussing or adopting the extraction of the collinear part of the Sivers distribution, $\Delta^{N} f_{q / p^{\uparrow}}(x)$, the collinear part of the Collins distribution, $\Delta^{N} D_{h / q^{\uparrow}}(z)$, or the transversity distribution, $h_{1}^{q}(x)$.

In particular, since equally good fits of the Sivers SIDIS asymmetry can be obtained with considerably different values of $\xi_{1}$, the extraction of the corresponding collinear part of the Sivers function, $\Delta^{N} f_{q / p^{\uparrow}}(x)$, or, equivalently, the prediction of the Sivers asymmetry in DY processes, may vary by up to a factor of 2 . A correct prediction of the Sivers asymmetry in DY processes is of particular importance, because of the expected sign change of the Sivers function in SIDIS and DY processes, which remains to be accurately tested. It is also relevant for the phenomenological study of the TMD evolution of the Sivers distribution.

Concerning the extraction of the collinear component of the Collins function $\Delta^{N} D_{h / q^{\uparrow}}(z)$ and the transversity distribution $h_{1}^{q}(x)$ from SIDIS and $e^{+} e^{-}$data, the uncertainty on $\xi_{1}$ seems to have milder effects. In fact, the rescaling factors for these functions, when changing the values of the parameters without altering the quality of the fits, are not far from unity, as shown in Figs. 6, 8, and 9. Although our plots cover all mathematically allowed values of $\xi_{1}$ down to $\xi_{1}=0$, the physical value of this parameter is expected to be larger than approximately 0.15 [31].

A precise determination of the parameter $\xi_{1}=$ $\left\langle p_{\perp}^{2}\right\rangle /\left\langle k_{\perp}^{2}\right\rangle$, at least according to the kinematical configuration of our Gaussian parametrization, is of crucial importance for a better knowledge of the Collins, Sivers, and transversity distributions. This parameter enters in the studies of the SIDIS multiplicities and unpolarized cross sections, which therefore deserve much attention, both experimentally and phenomenologically. In general, the QCD analysis of the available data is a formidable task, due to the difficulties in the correct implementation of the full theoretical framework and the quality of the experimental results, as recently pointed out in Ref. [45]. New important data, helpful in this respect, might soon be available from JLab 12, COMPASS, and future EIC experiments, as well as from Belle, BABAR, and BESIII in the fragmentation sector.

\section{ACKNOWLEDGMENTS}

This work was partially supported by the U.S. Department of Energy under Contract No. DE-AC0506OR23177 and within the TMD Collaboration framework, and by the National Science Foundation under Contract No. PHY-1623454. 
[1] D. W. Sivers, Phys. Rev. D 41, 83 (1990).

[2] D. W. Sivers, Phys. Rev. D 43, 261 (1991).

[3] J. C. Collins, Nucl. Phys. B396, 161 (1993).

[4] A. Airapetian et al. (HERMES Collaboration), Phys. Rev. Lett. 103, 152002 (2009).

[5] C. Adolph et al. (COMPASS Collaboration), Phys. Lett. B 717, 383 (2012).

[6] K. Allada et al. (Jefferson Lab Hall A Collaboration), Phys. Rev. C 89, 042201 (2014).

[7] A. Airapetian et al. (HERMES Collaboration), Phys. Lett. B 693, 11 (2010).

[8] C. Adolph et al. (COMPASS Collaboration), Phys. Lett. B 717, 376 (2012).

[9] C. Adolph et al. (COMPASS Collaboration), Phys. Lett. B 744, 250 (2015).

[10] K. Abe et al. (Belle Collaboration), Phys. Rev. Lett. 96, 232002 (2006).

[11] R. Seidl et al. (Belle Collaboration), Phys. Rev. D 78, 032011 (2008).

[12] J. P. Lees et al. (BABAR Collaboration), Phys. Rev. D 92, 111101 (2015).

[13] J. P. Lees et al. (BABAR Collaboration), Phys. Rev. D 90, 052003 (2014).

[14] M. Ablikim et al. (BESIII Collaboration), Phys. Rev. Lett. 116, 042001 (2016).

[15] W. Vogelsang and F. Yuan, Phys. Rev. D 72, 054028 (2005).

[16] J. C. Collins, A. V. Efremov, K. Goeke, S. Menzel, A. Metz, and P. Schweitzer, Phys. Rev. D 73, 014021 (2006).

[17] M. Anselmino, M. Boglione, U. D’Alesio, A. Kotzinian, S. Melis, F. Murgia, A. Prokudin, and C. Türk, Eur. Phys. J. A 39, 89 (2009).

[18] M. Anselmino, M. Boglione, U. D’Alesio, A. Kotzinian, F. Murgia, A. Prokudin, and S. Melis, Nucl. Phys. B, Proc. Suppl. 191, 98 (2009).

[19] A. Bacchetta and M. Radici, Phys. Rev. Lett. 107, 212001 (2011).

[20] M. Anselmino, M. Boglione, U. D'Alesio, S. Melis, F. Murgia, and A. Prokudin, Phys. Rev. D 87, 094019 (2013).

[21] M. Anselmino, M. Boglione, U. D’Alesio, J. O. GonzalezHernandez, S. Melis, F. Murgia, and A. Prokudin, Phys. Rev. D 92, 114023 (2015).

[22] M. Anselmino, M. Boglione, U. D’Alesio, J. O. GonzalezHernandez, S. Melis, F. Murgia, and A. Prokudin, Phys. Rev. D 93, 034025 (2016).

[23] M. Anselmino, M. Boglione, U. D’Alesio, F. Murgia, and A. Prokudin, J. High Energy Phys. 04 (2017) 046.
[24] M. Anselmino, M. Boglione, U. D’Alesio, A. Kotzinian, F. Murgia, A. Prokudin, and C. Türk, Phys. Rev. D 75, 054032 (2007).

[25] A. Bacchetta and A. Prokudin, Nucl. Phys. B875, 536 (2013).

[26] A. Signori, A. Bacchetta, M. Radici, and G. Schnell, J. High Energy Phys. 11 (2013) 194.

[27] M. Anselmino, M. Boglione, J. O. Gonzalez-Hernandez, S. Melis, and A. Prokudin, J. High Energy Phys. 04 (2014) 005.

[28] M. G. Echevarria, A. Idilbi, Z.-B. Kang, and I. Vitev, Phys. Rev. D 89, 074013 (2014).

[29] U. D'Alesio, M. G. Echevarria, S. Melis, and I. Scimemi, J. High Energy Phys. 11 (2014) 098.

[30] Z.-B. Kang, A. Prokudin, P. Sun, and F. Yuan, Phys. Rev. D 93, 014009 (2016).

[31] A. Bacchetta, F. Delcarro, C. Pisano, M. Radici, and A. Signori, J. High Energy Phys. 06 (2017) 081.

[32] I. Scimemi and A. Vladimirov, Eur. Phys. J. C 78, 89 (2018).

[33] J. Collins, Foundations of Perturbative QCD (Cambridge University Press, Cambridge, England, 2011).

[34] S. M. Aybat, J. C. Collins, J.-W. Qiu, and T. C. Rogers, Phys. Rev. D 85, 034043 (2012).

[35] M. G. Echevarria, A. Idilbi, and I. Scimemi, J. High Energy Phys. 07 (2012) 002.

[36] M. G. Echevarria, A. Idilbi, and I. Scimemi, Phys. Lett. B 726, 795 (2013).

[37] M. G. Echevarria, A. Idilbi, and I. Scimemi, Phys. Rev. D 90, 014003 (2014).

[38] T. C. Rogers, Eur. Phys. J. A 52, 153 (2016).

[39] J. O. Gonzalez-Hernandez, T. C. Rogers, N. Sato, and B. Wang, arXiv:1808.04396.

[40] M. Anselmino, M. Boglione, U. D’Alesio, S. Melis, F. Murgia, E. R. Nocera, and A. Prokudin, Phys. Rev. D 83, 114019 (2011).

[41] A. Bacchetta, M. Diehl, K. Goeke, A. Metz, P. J. Mulders, and M. Schlegel, J. High Energy Phys. 02 (2007) 093.

[42] M. Anselmino, M. Boglione, U. D’Alesio, S. Melis, F. Murgia, and A. Prokudin, Phys. Rev. D 79, 054010 (2009).

[43] M. Anselmino, M. Boglione, U. D’Alesio, A. Kotzinian, F. Murgia, and A. Prokudin, Phys. Rev. D 71, 074006 (2005).

[44] M. Boglione, J. O. Gonzalez-Hernandez, and R. Taghavi, Phys. Lett. B 772, 78 (2017).

[45] M. Boglione, U. D'Alesio, C. Flore, and J. O. GonzalezHernandez, J. High Energy Phys. 07 (2018) 148. 